\title{
Molecular, Topographic, and Functional Organization of the Cerebellar Cortex: A Study with Combined Aldolase $\mathrm{C}$ and Olivocerebellar Labeling
}

\author{
Izumi Sugihara and Yoshikazu Shinoda \\ Department of Systems Neurophysiology, Tokyo Medical and Dental University, Graduate School of Medicine, Tokyo 113-8519, Japan
}

\begin{abstract}
Aldolase C (zebrin) expression in Purkinje cells reveals stripe-shaped compartments in the cerebellar cortex. However, it is not clear how these compartments are related to cerebellar functional localization. Therefore, we identified olivocerebellar projections to aldolase $\mathrm{C}$ compartments by labeling climbing fibers with biotinylated dextran injected into various small areas within the inferior olive in rats. Specific rostral and caudal aldolase $C$ compartments were linked in an orderly manner by common olivocerebellar projection across the rostrocaudal boundary on lobule VIc-crus Ib. Based on the localization of the olivary origins of projection to similar compartments, the compartments and olivocerebellar projections could be sorted into five groups: group I, positive compartments extending from the posterior lobe to the anterior lobe innervated by the principal olive and some neighboring areas; group II, positive compartments localized within the posterior lobe innervated by several medial subnuclei; group III, vermal and central negative compartments innervated by the centrocaudal medial accessory olive; group IV, negative and lightly positive compartments in the hemisphere and the rostral and caudal pars intermedia innervated by the dorsal accessory olive and some neighboring areas; group $V$, the flocculus and nodulus. The olivocerebellar topography within each group was simple and suggests an "orientation axis" within the concerned parts of the inferior olive. Furthermore, parts of the inferior olive in each group receive specific afferent inputs, indicating a close relationship between aldolase $\mathrm{C}$ compartments and functional localization. Thus, the five-group scheme we propose here may integrate the molecular, topographic, and functional organization of the cerebellum.
\end{abstract}

Key words: climbing fiber; inferior olive; cerebellum; biotinylated dextran amine; zebrin; rat

\section{Introduction}

Neuronal activities related to specific behaviors can be recorded from distinct areas in the cerebellar cortex, suggesting the presence of a fine functional map. However, it is not clear how the cerebellar cortex is organized to give such fine functional localization. The cerebellar cortex shows both transverse organization based on its lobulation and longitudinal organization in which three subdivisions, the vermis, pars intermedia, and hemisphere, were originally recognized (Brodal, 1981). The longitudinal organization has been further elaborated by the demonstration of zones A-D (Voogd, 1967; Groenewegen and Voogd, 1977; Buisseret-Delmas and Angaut, 1993) and even much narrower "microzones" (Andersson and Oscarsson, 1978), which are based on topographic corticonuclear Purkinje cell projection and olivocortical climbing fiber projection. Recent analyses have

Received May 20, 2004; revised Aug. 25, 2004; accepted Aug. 27, 2004.

This study was supported by the following: the Human Frontier Science Program (Y.S.); Grants-in-Aid for Scientific Research from the Japan Society for the Promotion of Science (I.S. and Y.S.); Grants-in-Aid for Scientific Research on Priority Areas from The Ministry of Education, Culture, Sports, Science and Technology, Japan (I.S. and Y.S.); a grant from the Japan Space Forum (Y.S.); and a grant of the 21st Center of Excellence Program. We thank Drs. Y. Kubo and T. Misaka for help in antibody development and Western blotting and Dr. E. Lang for reading this manuscript.

Correspondence should be addressed to Dr. Yoshikazu Shinoda, Department of Systems Neurophysiology, Tokyo Medical and Dental University, Graduate School of Medicine, 1-5-45 Yushima, Bunkyo-ku, Tokyo 113-8519, Japan. E-mail: yshinoda.phy1@tmd.ac.jp.

DOl:10.1523/JNEUROSCI.1961-04.2004

Copyright $\odot 2004$ Society for Neuroscience $\quad$ 0270-6474/04/248771-15\$15.00/0 shown that the axon of an inferior olivary neuron provides multiple climbing fibers to a single narrow longitudinal band-shaped area comparable in size to a cortical microzone and sends collaterals to a small area in the cerebellar nucleus (Sugihara et al., $1999,2001)$. In contrast, a single lateral reticular nucleus neuron branches to form multiple mossy fibers that project to several longitudinal band-shaped areas (Wu et al., 1999). Although these single-axon projection patterns are likely to be directly related to cerebellar functional localization, their organization across the cerebellum has not been addressed in previous studies.

In addition to axonal projections, the fundamental organization of the cerebellar cortex may be revealed in the stripe-shaped expression patterns of several marker molecules for Purkinje cell populations (Hawkes and Leclerc, 1987; Brochu et al., 1990; Bailly et al., 1995; Herrup and Kuemerle, 1997). Among these molecules is zebrin II (Brochu et al., 1990; Hawkes, 1997), which has been shown to be aldolase C (Ahn et al., 1994). Although several studies have shown parallels between zebrin compartments and the climbing and mossy fiber projection patterns in some areas of the cerebellar cortex (Gravel et al., 1987; Matsushita et al., 1991; Voogd and Ruigrok, 1997; Voogd et al., 2003), a systematic comparison throughout the cerebellar cortex has not yet been made. Thus, the significance of zebrin compartments in terms of cerebellar morphology and physiology is not yet clear.

In the present study, we first formed a comprehensive twodimensional map of the aldolase $\mathrm{C}$ compartments in the entire rat 
cerebellar cortex from serial sections. We then carefully mapped olivocerebellar climbing fibers, labeled by a small injection of an anterograde tracer into various areas of the inferior olive, onto the aldolase $\mathrm{C}$ map generated from the same rat. A small injection was expected to label adjacent olivary neurons tightly coupled electrotonically (Llinás et al., 1974) and functionally related. The topographic olivocerebellar projection pattern and aldolase $\mathrm{C}$ compartments were then compared to reveal the fundamental functional and morphological organization of the cerebellar cortex.

\section{Materials and Methods}

Preparation of polyclonal antibody against aldolase C. A peptide (CGAATEEFIKRAEMNGLAAQGKYE) that consisted of the amino acid sequence 322-344 of rat aldolase C (Mukai et al., 1991), plus a cysteine residue at the $\mathrm{N}$ terminus to facilitate conjugation to the carrier protein $\mathrm{KLH}$, was synthesized and purified. This peptide was used for immunization, an ELISA test, affinity purification of antisera, and a blocking test in immunohistochemistry. Two rabbits (69075 and 69076) were immunized by five subcutaneous injections of $0.2 \mathrm{mg}$ of the peptide conjugated with KLH at 2, 3, 2, and 2 week intervals. The rabbits were exsanguinated 2 weeks after the final boost. Crude antisera of both rabbits were affinity purified against the immobilized immunizing peptide. Some purified antibody of 69076 was conjugated with biotin. The above procedures were performed by Sawady Technologies (Itabashi, Tokyo, Japan).

Western blot analysis. To test the specificity of the affinity-purified antibody, the whole cerebellum was dissected out from a Long-Evans male adult rat and then homogenized and sonicated in ice-cold PBS plus a protease inhibitor mixture (Complete; Roche, Basel, Switzerland). Protein concentration was determined by the Lowry method. Samples of 30 $\mu \mathrm{g}$ of proteins were separated by electrophoresis on 7.5 and $12 \%$ SDSpolyacrylamide gels and electrotransferred to polyvinylidene difluoride membranes (Immobilon-P; Millipore, Billerica, MA). Immunoblots were probed with purified antibodies against aldolase C $(125-250 \mathrm{ng} / \mathrm{ml}$; $1 \mathrm{hr}$ at room temperature) in a blocking solution containing $5 \%$ nonfat skim milk (Difco, Detroit, MI) in $20 \mathrm{~mm}$ Tris $\mathrm{HCl}$, pH 8.0, $150 \mathrm{~mm} \mathrm{NaCl}$, and $0.05 \%$ Tween 20 and were subsequently treated with donkey antirabbit IgG horseradish peroxidase-conjugated secondary antibody $\mathrm{F}\left(\mathrm{ab}^{\prime}\right)_{2}$ fragment (1:2000 dilution; $1 \mathrm{hr}$ at room temperature; NA9340; Amersham Biosciences, Little Chalfont, Buckinghamshire, UK). Detection was performed using chemiluminescence of 4-chloro-1-naphthol (ECL plus RPN 2132; Amersham Biosciences) according to the manufacturer's instructions. The membranes were then exposed to instant and peel-apart film (667; Polaroid, Waltham, MA).

Tracer injection and histological procedure. Fifty-four and 10 LongEvans adult rats were used for anterograde and retrograde labeling of olivocerebellar projections by tracer injections into the inferior olive and cerebellar cortex, respectively, followed by secondary labeling of aldolase C. Twelve Long-Evans adult rats were used only for labeling aldolase C. All of the experimental animals in this study were treated according to the guiding principles for the care and use of animals in the field of physiological sciences of the Japanese Physiological Society $(2001,2002)$, and the experimental protocols were approved by the Institutional Animal Care and Use Committee of Tokyo Medical and Dental University (no. 0020238). The methods of anesthesia, surgery, and histological procedures were the same as those described previously (Sugihara et al., 1999, 2001). Biotinylated dextran amine (BDA; D-1956; 10,000 molecular weight; Molecular Probes, Eugene, OR) was pressure injected at various locations in the inferior olive $(\sim 0.004 \mu \mathrm{l}$ of $10 \%$ solution in saline) for anterograde labeling. Two injections were made in the left and right olive to facilitate the study by using nearly exclusively contralateral projection. The injected tracer spread to a diameter of $0.1-0.2 \mathrm{~mm}$. Serial frozen sections ( $80 \mu \mathrm{m}$ thick) were cut from the entire cerebellum and medulla horizontally in seven rats and coronally in the others. After the sections were incubated with biotinylated peroxidase-avidin complex (PK6100 Elite ABC kit; Vector Laboratories, Burlingame, CA), BDA was visualized in black by incubating samples with diaminobenzidine $(0.5 \mathrm{mg} / \mathrm{ml})$, glucose oxidase ( $0.01 \mathrm{mg} / \mathrm{ml}$; type II, G-6125; Sigma, St. Louis, MO), nickel ammonium sulfate $(2 \mathrm{mg} / \mathrm{ml})$, ammonium chloride $(4 \mathrm{mg} / \mathrm{ml})$, and
$\beta$-D (+)-glucose (2 mg/ml) in Tris buffer (50 mm, pH 7.4) for 30-60 min and washed with PBS. For retrograde labeling, larger amounts of BDA $(\sim 0.01-0.02 \mu \mathrm{l})$ were injected bilaterally into lobules IX, VII, VIII, and crus II. Histological procedures for retrograde labeling were the same as those for anterograde labeling.

Immunohistological labeling of aldolase $C$. Brain sections either after or without $\mathrm{BDA}$ labeling were incubated with anti-aldolase $\mathrm{C}$ antibody (10 $\mathrm{ng} / \mathrm{ml}$ purified 69076 or $20 \mathrm{ng} / \mathrm{ml}$ purified 69075) in $2 \%$ normal goat serum and PBS with $0.15 \%$ Triton X-100 (PBST) for $48-72 \mathrm{hr}$ and then incubated with biotinylated anti-rabbit IgG antibody (BA-1000; Vector Laboratories) in PBST with 2\% normal goat serum for 6-12 hr. In later experiments, these two steps were replaced by a single step of incubation with biotin-conjugated anti-aldolase $\mathrm{C}$ antibody $(320 \mathrm{ng} / \mathrm{ml} 69076)$ in PBST with normal rabbit serum for 48-72 hr. After being washed, the sections were incubated with biotinylated peroxidase-avidin complex (PK6100 Elite ABC kit; Vector Laboratories) for 4-8 hr. When BDA labeling was performed, the sections were then incubated without heavy metal ions to stain the diaminobenzidine reaction product a brownish color in contrast to the black BDA reaction product. For experiments in which no BDA injection was made, the incubation solution contained nickel ammonium sulfate $(200 \mathrm{mg} / \mathrm{ml})$ in Tris buffer $(50 \mathrm{~mm})$ to stain the diaminobenzidine reaction product black. Sections were mounted on glass slides. Some sections were counterstained with thionine. Sections were coverslipped with Permount (Fisher Scientific, Fair Lawn, NJ). Stained axons and labeled aldolase $\mathrm{C}$ zones were photographed using a digital camera (DP-50; Olympus, Tokyo, Japan) attached to a microscope (BX41; Olympus). Photographs were assembled using Photoshop LE software (Adobe, San Jose, CA). The software was used to adjust contrast and brightness, but no digital enhancements were applied.

Tracing the continuity of aldolase $C$ zones in serial sections. Coronal, horizontal, and parasagittal sections stained with anti-aldolase $\mathrm{C}$ antibody were photographed with a film scanner (Dimage AF-5000; Minolta, Osaka, Japan) at a resolution of 4800 pixels per inch. A graphics file of the first section was opened in the bottom layer of graphics software (Illustrator 10; Adobe). Lines were superimposed in the top layer on the borders of aldolase $\mathrm{C}$ compartments and cortical outlines. The graphics file of the second section was then opened in the intermediate layer. The position and orientation of the second section was adjusted to obtain a best fit to the drawing of the first section in the top layer. The continuity of the compartments could be reliably judged by carefully comparing their positions in two consecutive sections. This procedure was repeated throughout the cerebellum.

Mapping climbing fiber locations on the unfolded cerebellar cortex. Because almost all olivocerebellar axons project contralaterally (Sugihara et al., 1999), projections from the left and right injections were analyzed separately. Ipsilateral projections, encountered infrequently, were ignored. A previous standardized unfolded representation of the Purkinje cell layer of the whole cerebellar cortex (Sugihara et al., 2001) was modified by incorporating the aldolase $\mathrm{C}$ labeling pattern. The mediolateral distance for a given compartment was measured at the apex of each folium from the midline along the cerebellar surface. For the sake of simplicity, the changes in the mediolateral positions of the compartments attributable to foliation of the cerebellum were ignored. To plot labeled climbing fibers, the aldolase $\mathrm{C}$ compartment in which they were distributed was carefully identified by following it through serial sections. The relative mediolateral position of the labeled climbing fiber in a given aldolase $\mathrm{C}$ compartment and the relative rostrocaudal position of the fiber within the folium were then measured to plot the climbing fiber on the unfolded representation of the cerebellar cortex.

The procedure for mapping injection sites in the horizontal representation of the inferior olive from brains cut coronally has been described previously (Sugihara et al., 2001). In brains cut horizontally, the contour of the inferior olive, the cut end of the medulla, the midline, and the injection spots were drawn using a camera lucida apparatus from each of the serial sections. The drawings were superimposed on each other to identify the contour of the entire inferior olive and the positions of the injection sites. We used the nomenclature of the subnuclei of the inferior olive proposed by Ruigrok and Cella (1995). A solid representation of the inferior olive was produced from camera lucida drawings using three- 

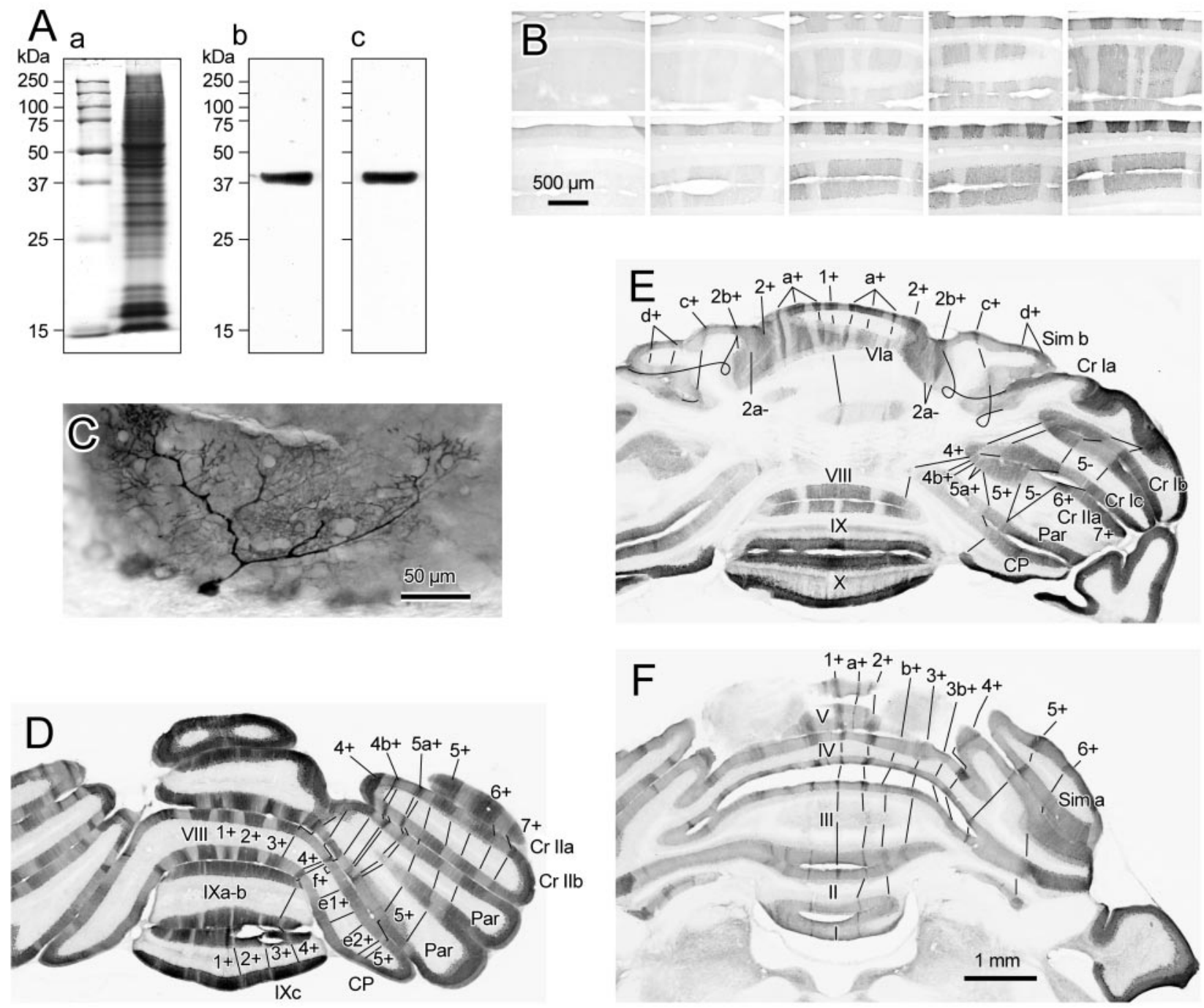

Figure 1. Specificity and labeling properties of anti-aldolase C antibodies. A, Western blots using antibodies against aldolase C. In comparing Coomasie staining of standard proteins (Prestained precision protein standards; $162-0372$; Bio-Rad, Hercules, $\mathrm{CA}$ ) and sample proteins of the cerebellum, a single identical band ( $39 \mathrm{kDa})$ was recognized in the Western blots of sample proteins by purified antibodies $69075(\mathrm{~b} ; 250 \mathrm{ng} / \mathrm{ml})$ and $69076(\mathrm{c} ; 125 \mathrm{ng} / \mathrm{ml})$. The results with 12\% SDS-polyacrylamide gel are shown here. B, Concentration-dependent blocking by the antigen peptide of immunohistological labeling of populations of Purkinje cells located in multiple zones in lobules VIII and IX. Adjacent coronal sections were incubated with one of the primary antibodies against aldolase C ( $20 \mathrm{ng} / \mathrm{ml} 69705$, top row; $10 \mathrm{ng} / \mathrm{ml} 69076$, bottom row) and various concentrations $(51.2,12.8,3.2,0.8$, and $0 \mu \mathrm{g} / \mathrm{ml}$; from left to right) of the peptide. C, High-magnification photomicrograph of a labeled Purkinje cell. The somata and dendrites as well as axons and axonal terminals are labeled by the anti-aldolase $C$ antibody. $D-F$, Photomicrographs of coronal sections of the cerebellum at the caudal $(D)$, intermediate $(E)$, and rostral $(F)$ levels labeled with the antibody 69076 . Lines show the continuity of positive compartments across folia. I-X, Lobules I-X; a- $c$, sublobules a-c; CP, copula pyramidis; $\mathrm{Cr}$ l, crus I of ansiform lobule; Crll, crus II of the ansiform lobule; sim, simple lobule.

dimensional graphics software (Rhinoceros; Robert McNeel \& Associates, Seattle, WA).

In our figures, all brain slices and drawings for which injections were made into the right inferior olive were reversed so that all climbing fibers were plotted in the right cerebellar cortex.

\section{Results}

Aldolase $\mathrm{C}$ antibodies

Antibodies were affinity purified from antisera obtained from two rabbits (69075 and 69076). These antisera and purified antibodies showed high reactivity in an ELISA test with the peptide. Western blotting of two purified antibodies showed that they specifically label a single identical band of protein with a molecular mass of $39 \mathrm{kDa}$ (Fig. $1 \mathrm{~A}$ ), similar to that of mouse aldolase $\mathrm{C}$ (36 kDa) (Ahn et al., 1994).

Histochemistry with these antisera and antibodies labeled a subpopulation of Purkinje cells located in the same pattern of multiple longitudinal compartments as with zebrin II antibody (Brochu et al., 1990) in all levels of the cerebellum (Fig. 1D-F). The addition of the antigen peptide to the solution of antialdolase $\mathrm{C}$ antibodies blocked the labeling in a concentrationdependent manner (Fig. $1 B$ ). Under high magnification, labeling was present in the somata, dendrites (Fig. 1C), axons, and axonal terminals (data not shown) of a population of Purkinje cells. This labeling was the same as that with zebrin II antibody (Brochu et al., 1990). These results indicated that the present two antialdolase $\mathrm{C}$ antibodies label the same compartments as zebrin II antibody, as expected from the fact that aldolase $\mathrm{C}$ is zebrin II (Ahn et al., 1994).

\section{Organization of reconstructed aldolase $\mathrm{C}$ compartments}

Because of the deep foliation of the cerebellar cortex, it is difficult to accurately determine the arrangement of the striped areas 
(“compartments") defined by the expression of aldolase C or any other molecular marker. Thus, complete reconstruction of the molecular compartments was accomplished only for the medial vermis (Doré et al., 1990; Ozol et al., 1999). For other areas of the cerebellum, data are available only for the superficial cortex (Hawkes and Leclerc, 1987; Brochu et al., 1990; Voogd et al., 2003; Voogd and Ruigrok, 2004), leaving the complete compartment pattern unidentified especially in the transitional region of the rostral and caudal cerebellum in simple lobule and crus I-II. Therefore, we carefully traced all aldolase C compartments through serial coronal sections (six rats) in the entire cerebellar cortex including the depths of the folia (see Materials and Methods). Because each compartment abruptly shifts laterally in the simple lobule and in crus Ia, compartments were also traced in horizontal (five rats) and parasagittal (one rat) sections for accuracy. Ignoring minor variations, the pattern of the major compartments was consistent among different individuals. Figure 2 shows a representative compartment pattern in the scheme of the unfolded cerebellar cortex.

We basically adopted the original numeric (Hawkes and Leclerc, 1987) and additional alphabetic nomenclatures of aldolase C compartments (Voogd et al., 2003; Voogd and Ruigrok, 2004). A plus sign was added for positive compartments. A negative compartment was designated by adding a minus sign to the name of the positive compartment located immediately medially to that negative compartment. To refer to a compartment, compartment $1+$ was abbreviated to $1+$, for example. Although the basic organization of aldolase C (zebrin II) compartments has been reported (Voogd et al., 2003; Voogd and Ruigrok, 2004), the additional characteristics of the aldolase C compartments revealed in the present study are described below.

The aldolase $\mathrm{C}$ compartments were not oriented exactly parallel to the midline, but instead ran in a laterocaudal direction in lobules I-VI and in a laterorostral direction in lobules VII-IX (Fig. 2). A hypothetical transverse line, designated as the "rostrocaudal boundary" (Fig. 2, dotted transverse line), could be drawn at the peak of the lateral expansion of positive compartments from caudal lobule VIc through crus Ib. At this line, all aldolase C-negative compartments disappeared, and the positive compartments merged into one transverse compartment. We use this boundary to define a "rostral" and a "caudal" cerebellar cortex. Although the major compartments in the rostral and caudal cerebellar cortex have been given the same numeric names $(1+, 2+$, and so on) (Hawkes and Leclerc, 1987), our map shows that they do not necessarily belong to the same continuous compartment (Fig. 2).

In the rostral cerebellar cortex, aldolase C-positive compartments could be classified into three types. The first type included those that were heavily labeled and continuous nearly throughout the rostral cerebellar cortex $(1+, 2+, 4+, 5+$, and $6+)$. The second type included those that were also heavily labeled but located mainly caudal to the primary fissure (Fig. 2, pf) $(\mathrm{a}+, 2 \mathrm{~b}+$, $\mathrm{c}+$, and $\mathrm{d}+)$. The third type included lightly labeled compartments (Fig. $1 F$ ) located mainly rostral to the primary fissure $(b+$, $3+$, and $3 \mathrm{~b}+)$. Among these positive compartments, $2 \mathrm{~b}+$ and $3 \mathrm{~b}+$ were newly defined and temporarily designated as such in this study. The most lateral part of original $2+$ in lobule VIa-c $(2 \mathrm{~b}+)$ was distinguished from $2+$ because it was separated from $2+$ by a narrow negative compartment (designated here as $2 \mathrm{a}-$ ) and $2+$ and $2 b+$ received different projections, as described later here. Compartment $3 \mathrm{~b}+$ was recognized between $3+$ and $4+$ in lobules IV-V (Fig. $1 F$ ). An additional positive compartment often seen between $\mathrm{d}+$ and $4+$ was also regarded as $\mathrm{d}+$ here (Fig. $1 E)$. Although a faintly labeled compartment was seen between

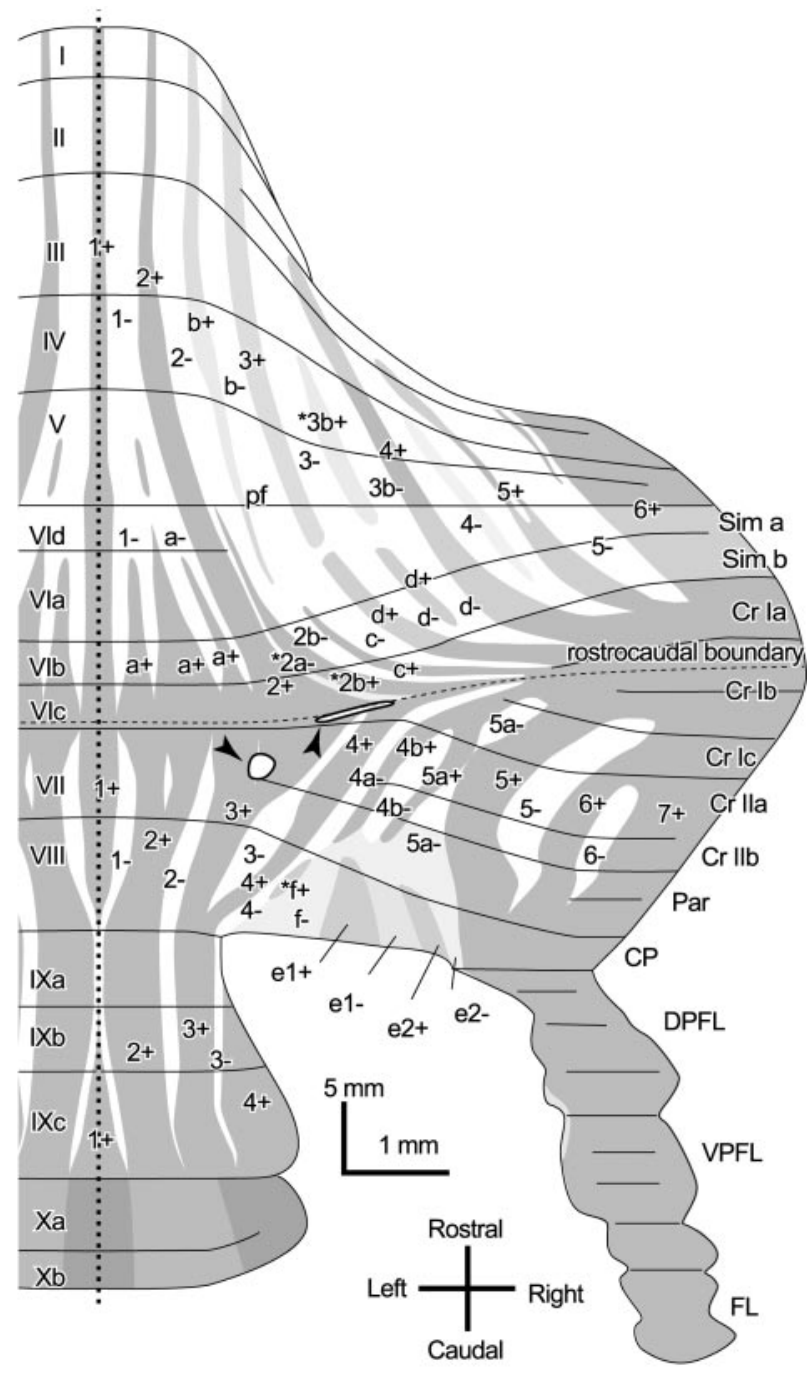

Figure 2. Reconstruction of aldolase C compartments throughout the cerebellar cortex. Continuity of the compartments in the rostrocaudal direction was determined by accurate tracing of individual compartments on serial sections and then plotted on a map of the unfolded cerebellar cortex (see Materials and Methods). Several new compartments were discovered (marked by asterisks prefixed to their proposed names) beyond those identified previously by Hawkes and Leclerc (1987), Voogd et al. (2003), and Voogd and Ruigrok (2004). A positive compartment and its laterally neighboring negative compartment usually have the same name with different suffixes ( + , positive compartment; - , negative compartment). The intensity of the gray color approximately corresponds to the intensity of labeling. The dotted line on the left indicates the rostrocaudal boundary of the compartment pattern (see Results). Arrowheads indicate areas in which the molecular layer is absent, one of which in the caudal apex of lobule VIc-crus I approximately coincided with the rostrocaudal border. I-X, Lobules I-X; $a-C$, sublobules $a-C ; C P$, copula pyramidis; Cr I, crus I of ansiform lobule; Cr II, crus II of ansiform lobule; DPFL, dorsal paraflocculus; FL, flocculus; Par, paramedian lobule; pf, primary fissure; Sim, simple lobule; VPFL, ventral paraflocculus.

$4+$ and $5+$ (not specifically designated), all the area between rostral $4+$ and rostral $5+$ was regarded as rostral $4-$ in this study. All labeling tended to be slightly weaker in the simple lobule than in other areas.

In the caudal cerebellar cortex, $4 \mathrm{~b}+$ and $5 \mathrm{a}+$ ended in the rostral paramedian lobule, and lightly labeled compartments $(\mathrm{e}+)$ (Voogd et al., 2003) were seen in the caudal paramedian lobule and copula pyramidis between $4+$ and $5+$. In this area, we temporarily divided the original e + into $\mathrm{e} 1+, \mathrm{e} 1-$, and $\mathrm{e} 2+$, because the center of $\mathrm{e}+$ was slightly more faintly labeled than the medial and lateral parts (Fig. $1 D$ ) and the olivocerebellar projec- 

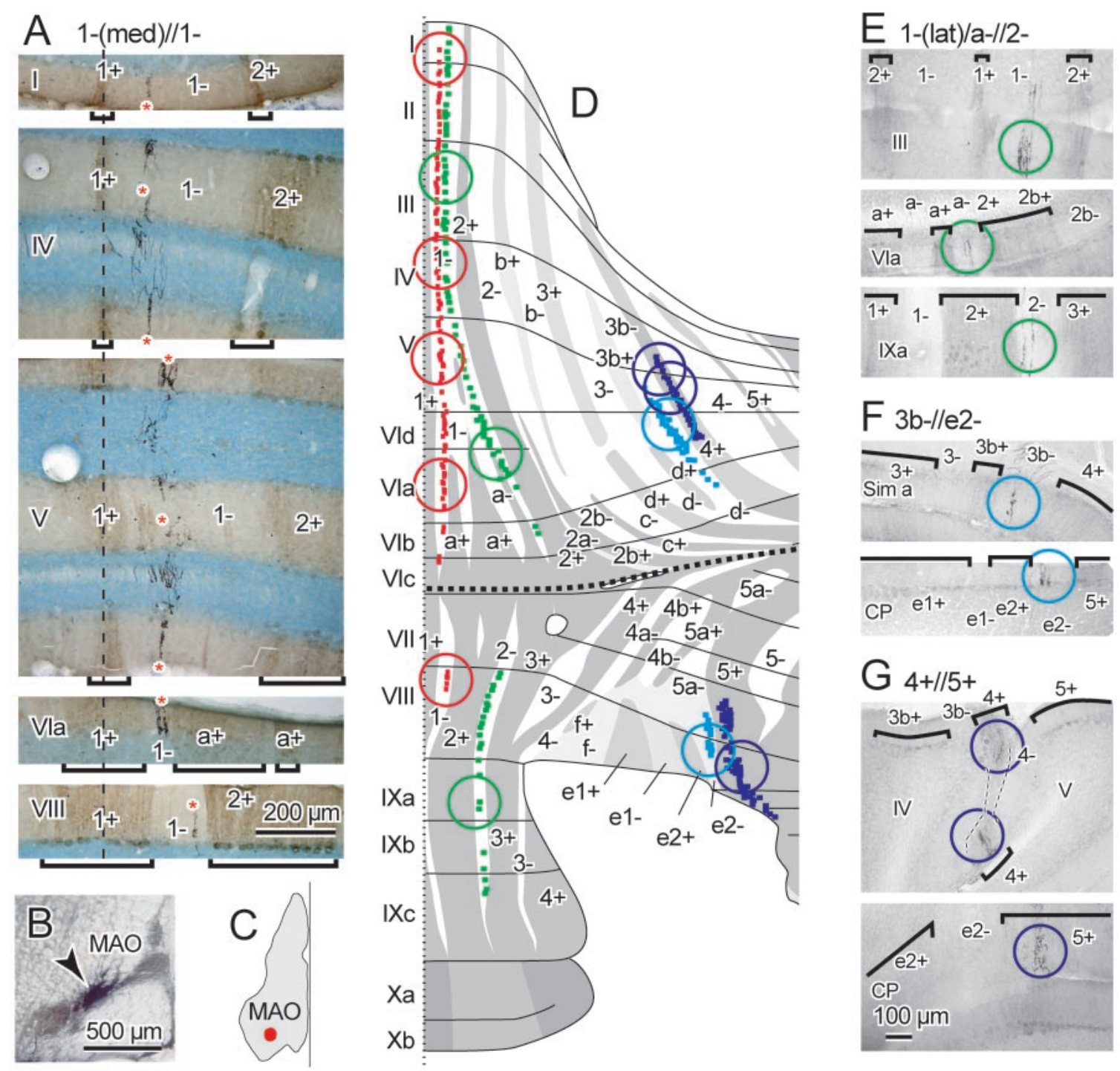

\section{$\mathrm{F} 3 \mathrm{~b}-1 / \mathrm{e} 2$}
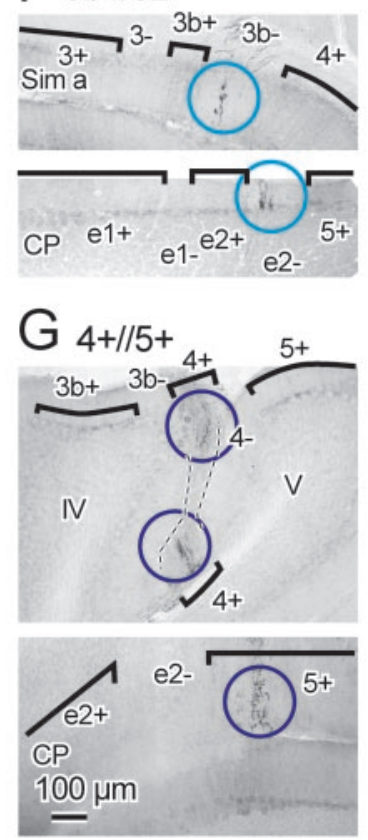

Figure 3. Narrow band-shaped longitudinal olivocerebellar projection connects corresponding rostral and caudal aldolase C compartments. A, Photomicrographs of several coronal sections showing that the narrow band of the distribution of labeled climbing fibers (asterisks) is parallel with and belongs to an aldolase C compartment. The brackets in each panel indicate positive compartments. All labeled climbing fibers were located in the medial portion of rostral compartment $1-$ or caudal compartment $1-$, hereafter classified as $1-$ (med)//1-(see Results). B, $C$, Photomicrograph $(B)$ and plot of the injection site in the inferior olive (C; horizontal representation of the left inferior olive) (the same experiment as shown in $A)$. D, Distribution of all labeled climbing fibers in the experiment shown in $A$ (red) and three other experiments (green, blue, violet) plotted in an unfolded representation of the cerebellar cortex. The colored squares indicate locations of individual labeled climbing fibers. The colored circles indicate the approximate areas of the photographs in $A, E, F$, and $G$. The dotted transverse line indicates the rostrocaudal boundary. $E-G$, Photomicrographs of double staining of aldolase $C$ and climbing fibers in the three experiments plotted in green, blue, and violet in $D$. Labeled climbing fibers are indicated by circles, the colors of which correspond to those in $D$. The injection sites of these experiments were located in the lateral subnucleus b of the caudal part of the MAO (c-MAO; $E)$, the rostral subnucleus b of the $c-M A O(F)$, and the rostral part of the MAO (r-MAO; G). I-X, Lobules I-X; a-c, sublobules a- $c$; $C P$, copula pyramidis; Sim, simple lobule.

tion pattern fit better with these smaller divisions (see below). A lightly labeled narrow compartment (designated $\mathrm{f}+$ ) was recognized immediately lateral to $4+$ with a narrow gap of a negative area $(4-)$ in lobule VIII-copula pyramidis (Fig. 1D). The original compartment $4-$ in crus I, II, and the rostral paramedian lobule seemed to be distinct from $4-$ in the caudal paramedian lobule and copula pyramidis in terms of olivocerebellar projection (see below). Therefore, the former was temporarily designated as $4 \mathrm{a}-$, because it was medial to $4 \mathrm{~b}+$.

\section{A specific pair of aldolase $\mathrm{C}$ compartments in the rostral and caudal cerebellum is linked through olivocerebellar projection}

To compare the olivocerebellar projection patterns with aldolase C compartments, we labeled climbing fibers by small injections of
BDA into individual subnuclei of the inferior olive (unilateral inferior olive injections in 23 animals and bilateral injections in 31 animals) and mapped the labeled climbing fibers to the identified aldolase $\mathrm{C}$ compartments. The diameter of spread from an injection site was $\sim 0.2 \mathrm{~mm}$ (Fig. $3 B, C$ ) and usually resulted in labeled climbing fibers being distributed to one or more segments of a single narrow longitudinal band with no $(n=31$ of 85 injections $)$ or $<10 \%(n=22)$ of labeled climbing fibers located outside the main band. We initially used the results from these experiments while simply ignoring the "outside" climbing fibers. For the remaining injections $(n=32),>10 \%$ of labeled climbing fibers were located outside the main termination band. However, the banding patterns from these outside distributions could usually be explained by spread of the tracer into adjacent but distinct areas in the inferior olive and correspondence of the aldolase $\mathrm{C}$ 
compartment-olivocerebellar band as determined from the more-limited injections.

In general, the bands of the labeled climbing fiber distribution were much narrower than a single aldolase C-positive or -negative compartment (Fig. 3A). Only in very narrow compartments, such as $2 \mathrm{a}-$, did the width of a band of labeled climbing fibers coincide with the width of the aldolase $\mathrm{C}$ compartment. However, the lateral borders of the aldolase $\mathrm{C}$ compartment and the band of the climbing fiber distribution were completely parallel (Fig. $3 A, D$ ).

Although the aldolase $\mathrm{C}$ compartment pattern was interrupted at the rostrocaudal boundary (above), a longitudinal band of labeled climbing fibers arising from a single injection site was distributed in both the rostral and caudal cerebellar cortex in almost all injections $(n=82$ of 85$)$ (Fig. $3 D-G)$, as reported previously (Sugihara et al., 2001). Figure 3 shows typical examples of individual longitudinal bands extending in both the rostral and caudal cerebellar cortex. Climbing fibers were distributed in the medial portion of rostral $1-$ in lobules I-VI and caudal $1-$ in lobule VII (Fig. 3D, red), the lateral portion of rostral $1-$ and caudal $2-$ (Fig. $3 D$, green; $E$ ), (rostral) $3 \mathrm{~b}-$ and (caudal) e2- (Fig. 3D, blue; $F$ ), and rostral $4+$ and caudal $5+$ (Fig. $3 D$, violet; $G$ ). Each band was distributed in a specific pair of rostral and caudal compartments positioned approximately at similar mediolateral distances from the midline. In some cases, the band of labeled climbing fibers was continuous from the rostral to caudal cerebellum across the rostrocaudal boundary, indicating the continuity of the specific rostral- and caudalpositive compartments.

Single olivocerebellar axons often innervate the rostral and caudal cerebellum by their axon collaterals (Sugihara et al., 2001). Taking advantage of this property, we linked a specific pair of rostral and caudal compartments based on common olivocerebellar projection. Here, we designate each pair of rostrocaudally corresponding compartments by using a format in which the names of the rostral and caudal compartments linked by the common olivocerebellar projection are separated by a double slash (i.e., rostral compartment//caudal compartment) (Table 1). Every pair of linked rostral and caudal compartments will be described below in relation to the common topographic olivocerebellar projection to the pair of compartments.

Whereas previous studies have presumed that rostral and caudal compartments with the same numbers corresponded to each other (Hawkes and Leclerc, 1987; Brochu et al., 1990), this relationship does not always hold. Compartment a+ (Voogd et al., 2003; Voogd and Ruigrok, 2004), located lateral to 1-(med)//1and caudal to the primary fissure, has been ignored in the original numeral nomenclature (Hawkes and Leclerc, 1987). This compartment was linked with caudal $2+(\mathrm{a}+/ / 2+)$. Accordingly, each of the other rostral numeric compartments was basically linked with a corresponding caudal compartment that was one digit higher $(2+/ / 3+$, $4+/ / 5+, 5+/ / 6+$, and $6+/ / 7+)$. Thus, the results in the present study can explain why the most lateral compartments were designated differently $[6+$ and $7+$ for the rostral and caudal compartments, respectively (Hawkes and Leclerc, 1987)].

\section{Relationship between aldolase $\mathrm{C}$ compartments and olivocerebellar topography}

We tried to identify the topographic relationship between subnuclei of the inferior olive and aldolase $\mathrm{C}$ compartments by examining the mapped distribution of climbing fibers labeled by an injection localized in a subnucleus. Additionally, to confirm the origin of the projection to some compartments in which not
Table 1. Link between rostral and caudal aldolase ( compartments determined by the major olivocerebellar projection pattern

\begin{tabular}{|c|c|c|}
\hline Rostral compartment & Caudal compartment & Designation for the pair ${ }^{a}$ \\
\hline $1+$ & $1+$ & $1+/ / 1+$ \\
\hline Medial 1- & $1-$ & $1-$ (med)//1- \\
\hline$a+$ & $2+$ & $a+/ / 2+$ \\
\hline Lateral 1- & $2-$ & $1-$ (lat)/a-//2- \\
\hline $2+$ & $3+$ & $2+/ / 3+$ \\
\hline $2 a-$ & $3-$ & $2 a-/ / 3-$ \\
\hline $2-^{b}$ & $4-^{c}$ & $2-/ / 4-$ \\
\hline $\mathrm{B}+{ }^{b}$ & $f+c$ & $\mathrm{~b}+/ / \mathrm{f}+$ \\
\hline $\mathrm{b}-{ }^{b}$ & $f-c$ & $\mathrm{~b}-/ / \mathrm{f}-$ \\
\hline $3+^{b}$ & $\mathrm{e} 1+^{c}$ & $3+/ / \mathrm{e} 1+$ \\
\hline $3-b$ & $\mathrm{e} 1-^{c}$ & $3-/ / \mathrm{e} 1-$ \\
\hline $3 b+b$ & $e 2+^{c}$ & $3 b+/ / e 2+$ \\
\hline $2 b+$ & $4+$ & $2 \mathrm{~b}+/ / 4+$ \\
\hline $2 b-$ & $4 a-$ & $2 b-/ / 4 a-$ \\
\hline$c+$ & $4 b+$ & $c+/ / 4 b+$ \\
\hline$c-$ & $4 b-$ & $c-/ / 4 b-$ \\
\hline$d+$ & $5 a+$ & $d+/ / 5 a+$ \\
\hline$d-$ & $5 a-$ & $d-/ / 5 a-$ \\
\hline $3 b-$ & e2- & $3 b-/ / e 2-$ \\
\hline $4+$ & $5+$ & $4+/ / 5+$ \\
\hline $4-$ & $5-$ & $4-/ / 5-$ \\
\hline $5+$ & $6+$ & $5+/ / 6+$ \\
\hline $5-$ & $6-$ & $5-/ / 6-$ \\
\hline $6+$ & $7+$ & $6+/ / 7+$ \\
\hline
\end{tabular}

${ }^{a}$ This format is used to indicate not only the linked compartments but also the olivocerebellar projection pattern to the compartments in this study.

${ }^{b}$ Compartments in lobules I-V.

'Compartments in the caudal paramedian lobule and copula pyramidis.

many climbing fibers happened to be labeled, retrograde tracer was injected into these compartments $(n=10)$ and the locations of retrogradely labeled neurons within the inferior olive were determined. In this study, we used the conventional nomenclature of the olivary subnuclei in which the inferior olive is subdivided into three major subnuclei [principal olive (PO), dorsal accessory olive (DAO), and medial accessory olive (MAO)] and four small subnuclei [subnucleus $\beta$, dorsomedial cell column (DMCC), dorsal cap (DC), and ventrolateral outgrowth (VLO)]. The PO is further subdivided into the ventral and dorsal lamellas (v-PO, d-PO) and the dorsomedial group (DM), the DAO is divided into dorsal and ventral folds (d-DAO, v-DAO), and the MAO is divided into rostral and caudal parts ( $\mathrm{r}-\mathrm{MAO}, \mathrm{c}-\mathrm{MAO}$ ) (Ruigrok and Cella, 1995). The c-MAO was further subdivided cytologically into lateral, intermediate, and medial parts (subnucleus a, b, and c, respectively) (Gwyn et al., 1977).

We noticed that origins of the projections to the negative and lightly labeled positive compartments are clustered only in two areas of the inferior olive: (1) the subnucleus b of the c-MAO; and (2) neighboring dorsal subnuclei including the d-DAO, v-DAO, and central and rostral DM. All the rest of the inferior olive projected to the darkly labeled positive compartments. Furthermore, concerning the projections to the darkly labeled positive compartments, mainly lateral areas of the inferior olive (subnucleus a, $\mathrm{r}-\mathrm{MAO}$, most of the $\mathrm{v}-\mathrm{PO}$, and d-PO) projected to the compartments that extend beyond the primary fissure to the anterior lobe (designated as group I), whereas mainly medial areas of the inferior olive (subnucleus $\mathrm{c}$, subnucleus $\beta$, DMCC, caudal DM, and caudomedial v-PO) projected to the compartments that do not extend beyond the primary fissure but are located in the posterior lobe (group II). In addition, the projection from the DC and VLO seemed distinct from the others (group V). Concerning the pro- 

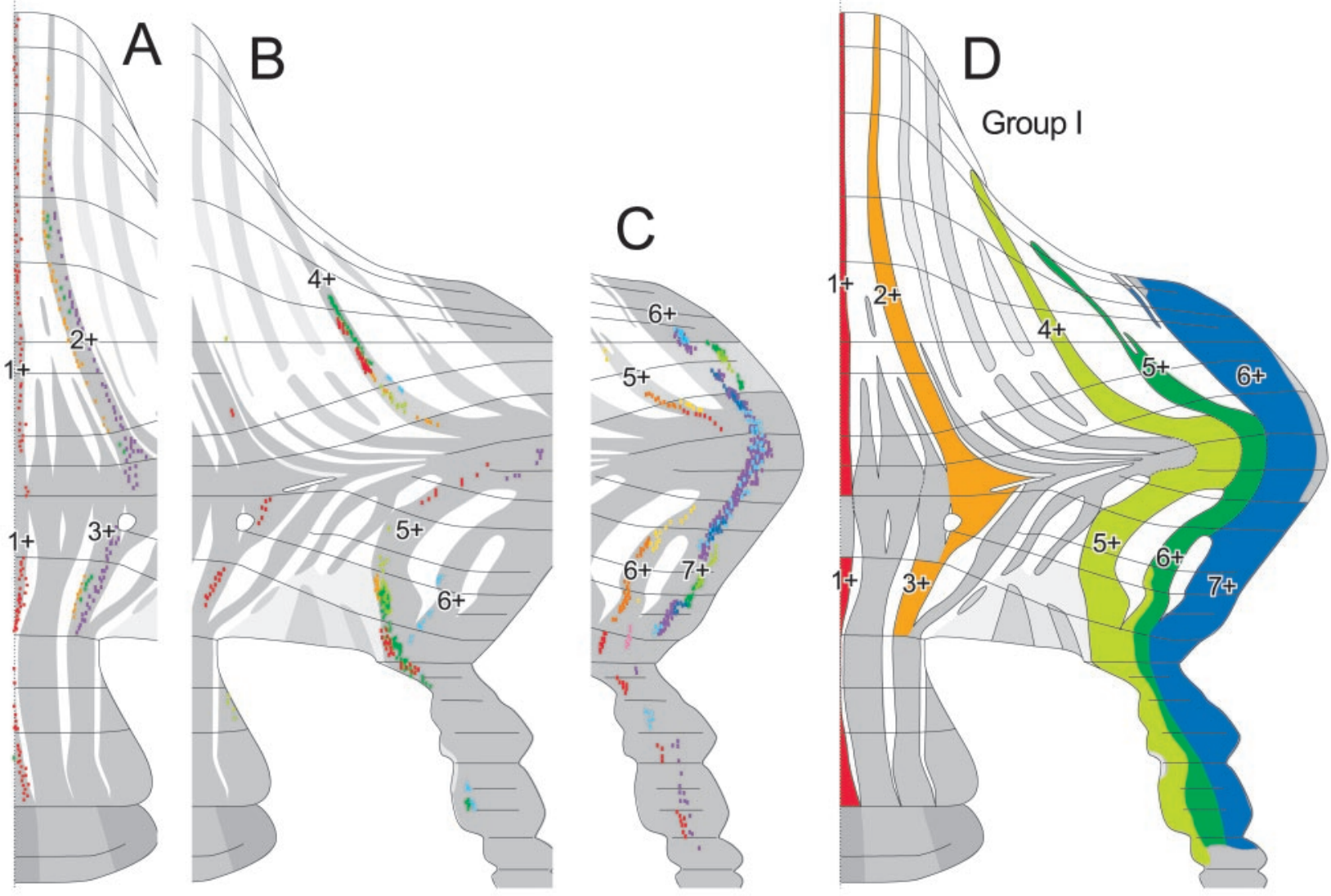

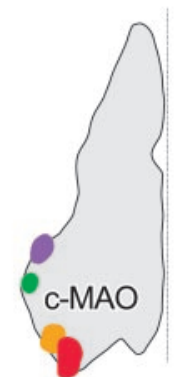

Dorsal view

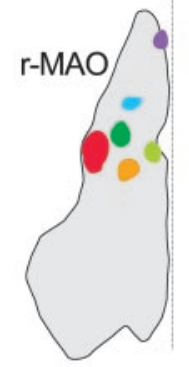

Dorsal view
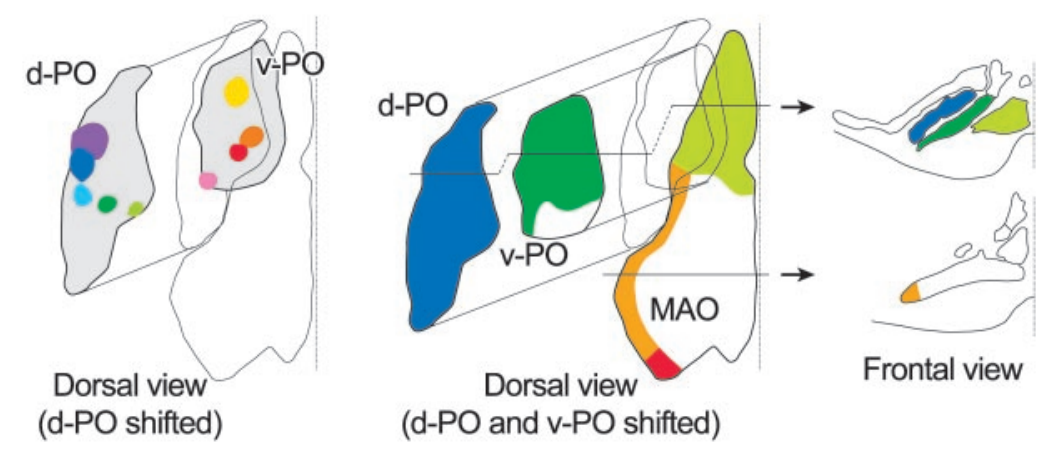

Figure 4. Mapping of labeled climbing fibers and summarized topographic scheme of the olivocerebellar projection belonging to group I. This group consisted of projections from caudal subnucleus $a$ and the intermediate and rostral subnucleus a of the caudal part of the MAO (c-MA0) to $1+/ / 1+$ and $2+/ / 3+$, respectively, from the rostral part of the MA0 (r-MA0) to $4+/ / 5+$; from the ventral lamella of the PO (v-P0), except for the caudomedial part, to $5+/ / 6+$; and from the dorsal lamella of the P0 (d-P0) to $6+/ / 7+$. $A-C$, Plots of labeled climbing fibers in 19 injections terminating in $1+/ / 1+$ and $2+/ / 3+(A), 4+/ / 5+(B)$, and $5+/ / 6+$ and $6+/ / 7+(C)$. Labeled climbing fibers in all of these experiments $(A-C)$ were nearly aligned in a single narrow band in a pair of linked rostral and caudal aldolase ( compartments, except for one case ( $B$; red), in which some outside climbing fibers were seen. $D$, Putative olivocerebellar topography within group I.

jections to the negative and lightly labeled positive compartments, the subnucleus b (group III) and the neighboring dorsal subnuclei (group IV) mostly projected to distinct compartments in the cerebellar cortex (medial and lateral, respectively). The above indicated that the olivocerebellar projection to aldolase $\mathrm{C}$ compartments could be classified into five groups based on the labeling and geometric similarity of the target compartments and the adjacency of the olivary subnuclei of origin. Therefore, we describe the projection patterns by sorting them into these five groups in the following sections. Group V, which consists of the projections to the flocculus and nodulus from the DC and VLO of the inferior olive (Sugihara et al., 2004), was not addressed in this study.

In previous studies, olivocerebellar projection patterns used to be sorted based on the subdivisions of the inferior olive. In- deed, relatively simple projections were seen in a few subnuclei in which one subnucleus exclusively innervated only one pair of aldolase C compartments: the r-MAO innervated $4+/ / 5$ and d-PO innervated $6+/ / 7+$. However, olivocerebellar projections were more complicated in other subnuclei. For example, some subnuclei (e.g., d-DAO and v-DAO) innervated multiple pairs of neighboring aldolase $\mathrm{C}$ compartments, and some other subnuclei (e.g., c-MAO, v-PO, and DM) innervated multiple pairs of noncontiguous aldolase $\mathrm{C}$ compartments, suggesting that these subnuclei were composed of parts with distinct projection patterns. Furthermore, parts of some different subnuclei innervated the same pair of compartments. Therefore, we did not use not the conventional subdivisions of the inferior olive but used the five groups that we proposed above to sort the results in the present study. 


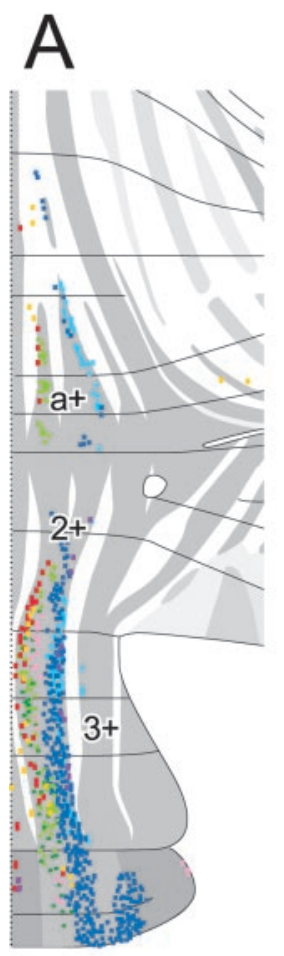

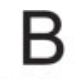
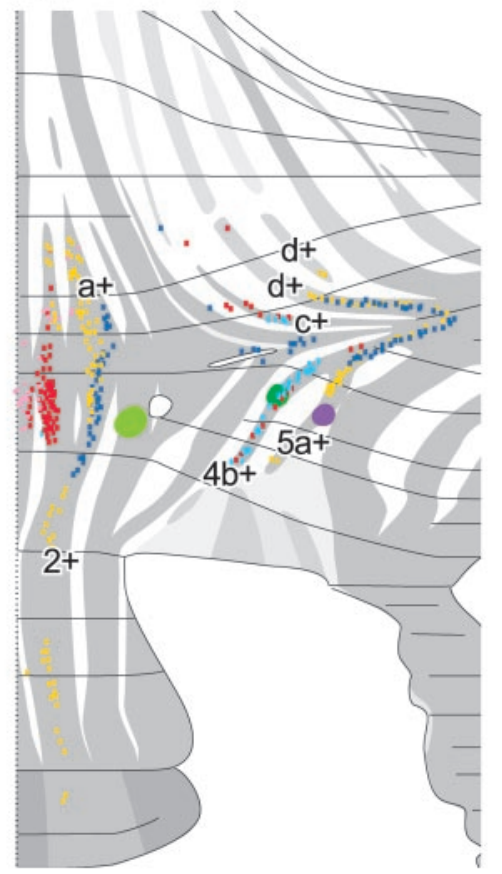

C
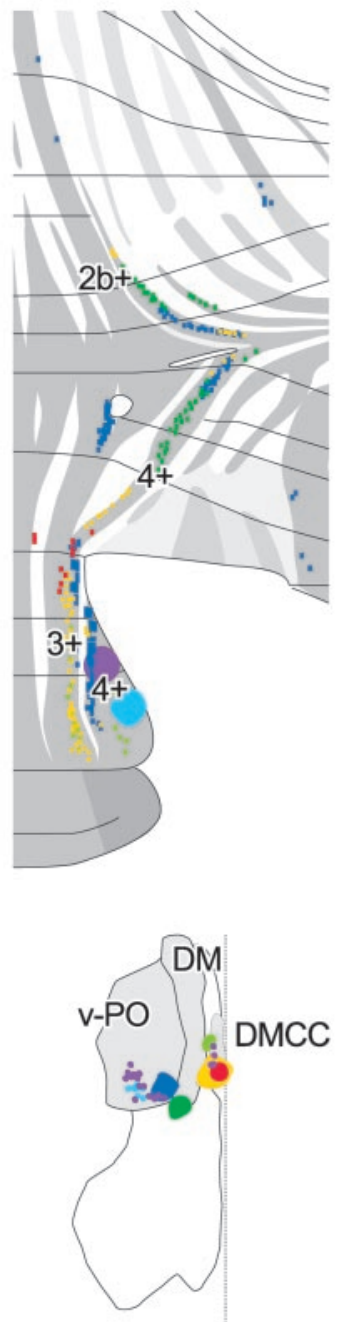

Dorsal view

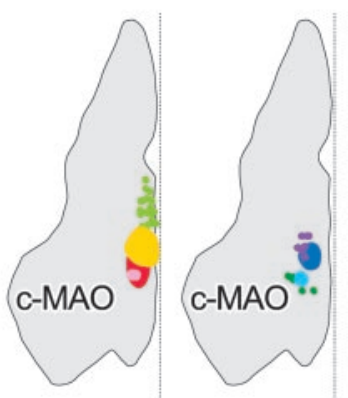

Dorsal view
Dorsal view (BETA shifted)
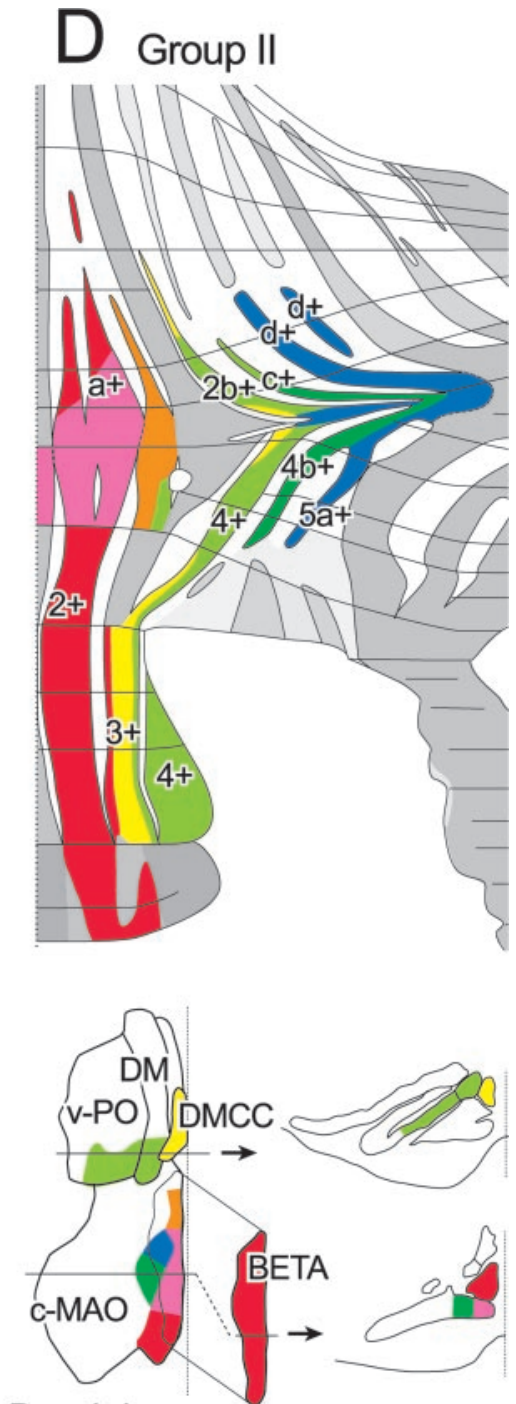

Dorsal view

(BETA shifted)

Frontal view

Figure 5. Mapping of labeled climbing fibers and summarized topographic scheme of the olivocerebellar projection belonging to group II. This group consisted of projections from subnucleus $\mathrm{C}$ of the caudal part of the MAO (c-MAO) to $\mathrm{a}+/ / 2+, \mathrm{c}+/ / 4 \mathrm{~b}+$, and $\mathrm{d}+/ / 5 \mathrm{a}+$; from subnucleus $\beta$ (BETA) to $\mathrm{a}+/ / 2+$; and from the DMCC, caudal dorsomedial subnucleus of the PO (DM), and caudomedial ventral lamella of the $\mathrm{PO}(\mathrm{v}-\mathrm{PO})$ to $2 \mathrm{~b}+/ / 4+$. A, Plots of climbing fibers in eight injections terminating in $\mathrm{a}+/ / 2+$ labeled with injections into the subnucleus $\beta$ and caudal subnucleus c of the c-MA0. Two injections (red and light green) were spread to both the c-MA0 and subnucleus $\beta . B$, Plots of climbing fibers in five injections terminating in a $+/ / 2+, c+/ / 4 b+$, and $d+/ / 5 a+$ and retrogradely labeled olivary neurons in three experiments (yellow green, green, and purple). Plots of injection sites and retrogradely labeled neurons in the inferior olive were classified into medial and lateral ones and drawn separately to improve visibility. The yellow injection was spread to the subnucleus $\beta$, presumably explaining the labeled climbing fibers in lobules VIII-IX in this case. C, Plots of climbing fibers in five injections terminating in $2 \mathrm{~b}+/ / 4+$ and retrogradely labeled olivary neurons in two experiments (light blue and purple circles). Labeled climbing fibers in each anterograde labeling experiment $(A, B)$ were nearly aligned in a single narrow band in a pair of linked rostral and caudal aldolase $C$ compartments with few, if any, outside climbing fibers, except for a few cases ( $A$, blue and light blue; $B$, blue) in which climbing fibers were distributed in multiple compartments. D, Putative olivocerebellar topography within group II.

\section{Group I}

Group I consisted of heavily labeled aldolase C-positive compartments in the anterior lobe (rostral $1+, 2+, 4+, 5+$, and $6+$ ) and their corresponding positive caudal compartments (caudal $1+$, $3+, 5+, 6+$, and $7+)$. The compartments in this group were innervated by neighboring central and lateral parts of subdivisions of the inferior olive.

The caudal pole of the most lateral part of the c-MAO (subnucleus a) projected to rostral $1+$ from lobules I to VIc and caudal $1+$ from VIII to IXc (Fig. $4 \mathrm{~A}$, red). This projection pattern was simply designated as $1+/ / 1+$ because it covers most of the linked compartments $1+/ / 1+$. Although $1+$ is located on the midline, the labeled climbing fibers were distributed mostly on the side contralateral to the injection site, agreeing with the general contralateral olivocerebellar projection. The rest of subnucleus a, elongated in the rostrocaudal direction, projected to rostral $2+$ in lobules I through VIb and to caudal $3+$ in lobules VII and VIII $(2+/ / 3+)$ (Fig. 4A; yellow, green, and purple). The rostral and central subnucleus a projected laterally and medially within compartments $2+/ / 3+$, respectively.

The r-MAO projected to rostral $4+$, caudal $5+$, and its putative extension in the paraflocculus and flocculus and the most medial portion of the caudal $6+$ in the paramedian lobule (Fig. $4 B)$. This projection pattern was simply designated as $4+/ / 5+$ because the involvement of caudal $6+$ was relatively small. The $\mathrm{v}-\mathrm{PO}$, except for its caudomedial portion (see below), projected 

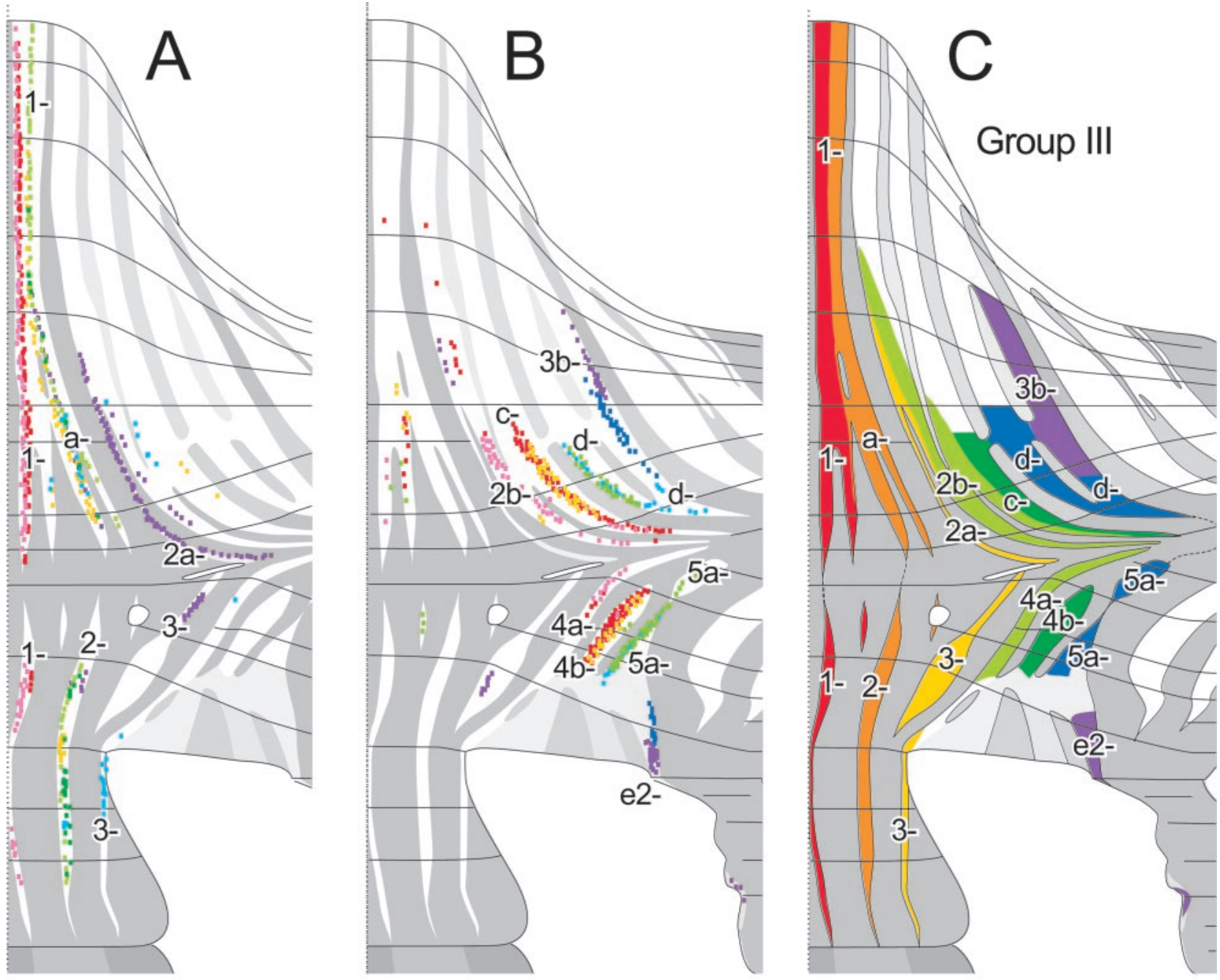

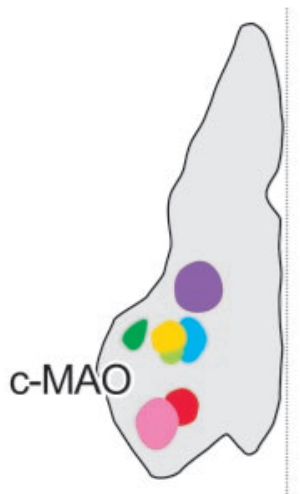

Dorsal view

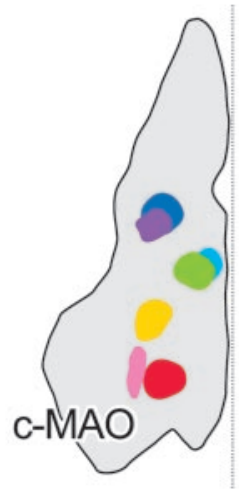

Dorsal view

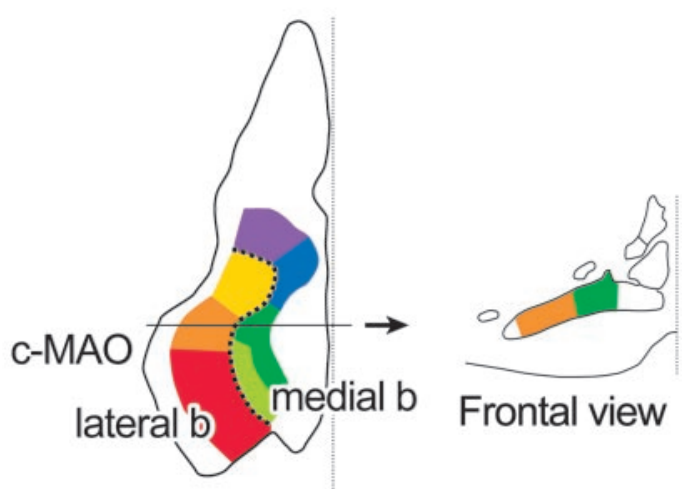

Dorsal view

Figure 6. Mapping of labeled climbing fibers and summarized topographic scheme of the olivocerebellar projection belonging to group III. This group consisted of projections from subnucleus $\mathrm{b}$ of the caudal part of the MAO (c-MA0) to $1-$ (med)//1-, $1-$ (lat)/a $-/ / 2-, 2 \mathrm{a}-/ / 3-, 2 \mathrm{~b}-/ / 4 \mathrm{a}-, \mathrm{c}-/ / 4 \mathrm{~b}-, \mathrm{d}-/ / 5 \mathrm{a}-$, and $3 \mathrm{~b}-/ / \mathrm{e} 2-$. $A$, Plots of labeled climbing fibers in seven injections terminating in $1-$ (med) $/ / 1-, 1-($ lat $) / a-/ / 2-$, and $2 a-/ / 3-$. B, Plots of labeled climbing fibers in seven injections terminating in $2 b-/ / 4 a-, c-/ / 5 a-, d-/ / 5 a-$, and $3 \mathrm{~b}-/ / \mathrm{e} 2-$. Labeled climbing fibers in each anterograde injection in $A$ and $B$ were nearly aligned in a single narrow band with few, if any, outside climbing fibers. $C$, Putative olivocerebellar topography within group III determined from the results shown in $A$ and $B$ and 13 other cases. $b$, Subnucleus $b$.

to $5+/ / 6+$ (Fig. $4 C$; red, orange, yellow, and pink). The d-PO projected to $6+/ / 7+$ (Fig. $4 C$; light green, green, light blue, blue, and purple). The topographic projection pattern of group I is summarized in Figure $4 \mathrm{D}$ based on the data obtained in 20 injections including the above.

\section{Group II}

Group II consisted of heavily labeled aldolase C-positive compartments that do not extend rostrally beyond the primary fissure $(\mathrm{a}+, 2 \mathrm{~b}+, \mathrm{c}+$, and $\mathrm{d}+)$ and their corresponding caudal compartments $(2+, 4+, 4 \mathrm{~b}+$, and $5 \mathrm{a}+)$. These compartments were 
innervated by several medial subnuclei and adjacent areas in the inferior olive (subnucleus c, subnucleus $\beta$, DMCC, caudal parts of the DM, and caudomedial part of the $\mathrm{v}-\mathrm{PO}$ ).

Subnucleus c, which is the most medial portion of the c-MAO, was divided into caudal, medial, and lateral parts. The caudal subnucleus $\mathrm{c}$ as well as the caudal subnucleus $\beta$ projected to the medial half of $\mathrm{a}+$ in lobules VIa-b and $2+$ in lobules VIII-IX (Fig. 5A; pink, red, yellow, light green, and green). The projections from the caudal subnucleus $\mathrm{c}$ and from the caudal subnucleus $\beta$ could not be distinguished. The medial subnucleus c projected to $\mathrm{a}+$ in VIb-c and $2+$ in VII and also to adjacent areas in caudal $1+$ and $3+$ [Fig. 5B; pink, red, parts of yellow, and light green (retrograde)].

The lateral subnucleus $\mathrm{c}$ was further subdivided based on the difference in the projection pattern. The caudal and rostral parts of lateral subnucleus $c$ mainly projected to $c+/ / 4 b+[$ Fig. $5 B$, light blue and green (retrograde)] and to $d+/ / 5 \mathrm{a}+[$ Fig. $5 B$, part of blue and violet (retrograde)], respectively. In anterograde labelings, the projections to $\mathrm{c}+/ / 4 \mathrm{~b}+($ or $\mathrm{d}+/ / 5 \mathrm{a}+)$ from the lateral subnucleus $\mathrm{c}$ and the projection to $\mathrm{a}+/ / 2+$ in lobules VI-VII from the medial subnucleus $\mathrm{c}$ were often contaminated to a variable extent by each other because of the adjacency of the medial and lateral subnucleus c (Fig. 5B; red, yellow, and blue). However, no single reconstructed axons originating from these areas of the inferior olive had transverse collaterals to innervate both the vermis and pars intermedia (Sugihara et al., 2001), supporting that the medial and lateral subnucleus $\mathrm{c}$ were distinct in the projection pattern.

The central and rostral subnucleus $\beta$ projected to the lateral half of $\mathrm{a}+$ in lobules VIa-b and $2+$ in lobules VIII-IX (Fig. 5A; light blue, blue, and purple) and to the most medial part of $3+$ in lobule IX (Fig. 5A, light blue). These projections as well as the projections from the caudal subnucleus $\beta$ and caudal and medial subnucleus c (above) were simply designated as $a+/ / 2+$.

The DMCC projected to the central and lateral portions of $3+$ in lobules IXa-c to $4+$ in lobule VIII and to some medial portions of rostral $2 \mathrm{~b}+$ and caudal $4+$ in crus I (Fig. $5 C$; red, yellow, and light green). The caudal part of the DM projected to rostral $2 \mathrm{~b}+$ in the simple lobule, to caudal $4+$ in crus II and the paramedian lobule, and to some $\mathrm{c}+$ in the simple lobule (Fig. $5 C$, green). The caudomedial $v$-PO projected to rostral $2 \mathrm{~b}+$ and caudal $4+$ in crus I and to $4+$ and the most lateral $3+$ in lobule IX [Fig. $5 C$; blue, purple, and light blue (retrograde)]. In summary, the DMCC, caudal DM, and caudomedial $v$-PO project to $2 \mathrm{~b}+/ / 4+$. Figure $5 D$ shows the complete topographic projection pattern of group II based on the data obtained in 26 injections including the above.

\section{Group III}

The central portion of the c-MAO (subnucleus b) projected to all of the aldolase C-negative compartments in the vermis and the central pars intermedia. Based on its topographic projection pattern, subnucleus b was divided into lateral and medial parts, each of which was further subdivided into three to four areas rostrocaudally (Fig. 6, inset).

The caudal, intermediate, and rostral parts of the lateral subnucleus b projected to $1-($ med $) / / 1-$ (Fig. 6A, pink and red), $1-$ (lat)/a-//2- (Fig. $6 A$; yellow, light green, green, and parts of light blue) and to $2 \mathrm{a}-/ / 3-$ (Fig. $6 \mathrm{~A}$, purple and parts of light blue), respectively.

The caudolateral, caudomedial, intermediate, and rostral parts of the medial subnucleus b projected to $2 \mathrm{~b}-/ / 4 \mathrm{a}-$ (Fig. $6 \mathrm{~B}$, pink), $\mathrm{c}-/ / 4 \mathrm{~b}-$ (Fig. $6 B$, red and yellow), $\mathrm{d}-/ / 5 \mathrm{a}-$ (Fig. $6 \mathrm{~B}$, green and light blue), and $3 \mathrm{~b}-/ / \mathrm{e} 2-$ (Fig. $6 B$, blue and parts of purple), respectively. The topo- graphic projection pattern of group III is summarized in Figure $6 C$ based on the above data and 13 other injections.

The projections to $2 \mathrm{a}-/ / 3-$ and $3 \mathrm{~b}-/ / \mathrm{e} 2-$ from the rostral parts of the lateral and medial subnucleus $b$ were separately labeled in three cases (Fig. $6 \mathrm{~A}$, purple and light blue; $B$, blue), indicating that they are mostly distinct. The labeling in both $3 \mathrm{~b}-/ / \mathrm{e} 2-$ and $2 \mathrm{~b}-/ / 3-$ in the purple case in Figure $6 \mathrm{~B}$ may be attributable to the spread of the tracer to the origins of the two projections, although we cannot exclude the possibility of collateralization between these areas in some axons.

\section{Group IV}

The lightly positive compartments and their neighboring negative compartments in the rostral and caudal portions of the pars intermedia $(2-, \mathrm{b}+, \mathrm{b}-, 3+, 3-$, and $3 \mathrm{~b}+; 4-, \mathrm{f}+, \mathrm{f}-, \mathrm{e} 1+, \mathrm{el}-$, and $\mathrm{e} 2+)$ and all of the negative compartments in the hemisphere (rostral 4- and 5- and caudal 5- and 6-) are innervated by several neighboring subnuclei of the dorsal inferior olive (d-DAO, $\mathrm{v}-\mathrm{DAO}$, and the central and rostral DM). Because olivocerebellar projection patterns to the lightly positive and negative compartments could not be distinguished clearly from each other, these compartments were temporarily classified together as group IV.

The olivocerebellar projection pattern in group IV was more complicated than those in the other groups. Especially, labeled climbing fibers originating from the $\mathrm{v}$-DAO were distributed in multiple aldolase $\mathrm{C}$ compartments in a few cases (Fig. $7 B$, blue and yellow), which may be related to the transverse branching of single olivocerebellar axons found in the presumably equivalent area of the cat cerebellum (Ekerot and Larson, 1982). Consequently, the topographic relationship of the v-DAO could not be fully understood based on the present results.

The medial and central parts of the d-DAO projected to 2-//4- (Fig. 7A; pink, red, orange and yellow, and part of light green). Lateral parts of the d-DAO and the caudolateral pole of the $\mathrm{v}$-DAO projected to $\mathrm{b}+/ / \mathrm{f}+$ and $3+/ / \mathrm{e} 1+$, probably respectively (Fig. 7A, green).

The lateral part of the $\mathrm{v}-\mathrm{DAO}$ projected to $\mathrm{b}-/ / \mathrm{f}-$ (Fig. $7 A$; part of light green, light blue, blue, and purple). It also projected to $2-$ in lobules I-III and to $3 \mathrm{~b}-$ in lobules II-III, although this projection was less dense than that to $\mathrm{b}-$ or $\mathrm{f}-$. The intermediate part of the v-DAO projected to $2-$ in the simple lobule to $3-$ in lobules IV-V, to $4-$ and the most medial part of 5- in lobules $\mathrm{I}-\mathrm{V}$, and to e1- in the copula pyramidis and its extension in the paramedian lobule (Fig. $7 B$; red, yellow, green, and blue), where $3-/ / e 1-$ may be regarded as the main projection area by analogy to other pairs (Fig. 7B, yellow). Although no climbing fibers were labeled in $3 \mathrm{~b}+$ or $\mathrm{e} 2+$, they may form a linked pair innervated by certain areas of the $\mathrm{v}-\mathrm{DAO}$, because $3 \mathrm{~b}+$ and $\mathrm{e} 2+$ were similar to and parallel with $3+$ and $\mathrm{e} 1+$, respectively.

The medial part of the $\mathrm{v}-\mathrm{DAO}$ projected mainly to $4-/ / 5-$ (Fig. 7C). It also projected to small areas of the medial edge of rostral 5- in lobule $\mathrm{V}$ and the simple lobule and to small areas on the lateral border of caudal $5 \mathrm{a}-$ in the paramedian lobule. The rostral and central DM projected to $5-/ / 6-$ (Fig. $7 D)$. It also projected to the lateral part of the caudal $5-$ in crus Ic and rostral crus IIa. These topographic projection patterns of group IV are summarized in Figure $7 E$.

\section{The topography of olivocerebellar projection can be explained by assuming a distinct "orientation axis" in each group}

We noticed a simple topographic principle in each of the olivocerebellar projection groups described above. The mediolateral direc- 

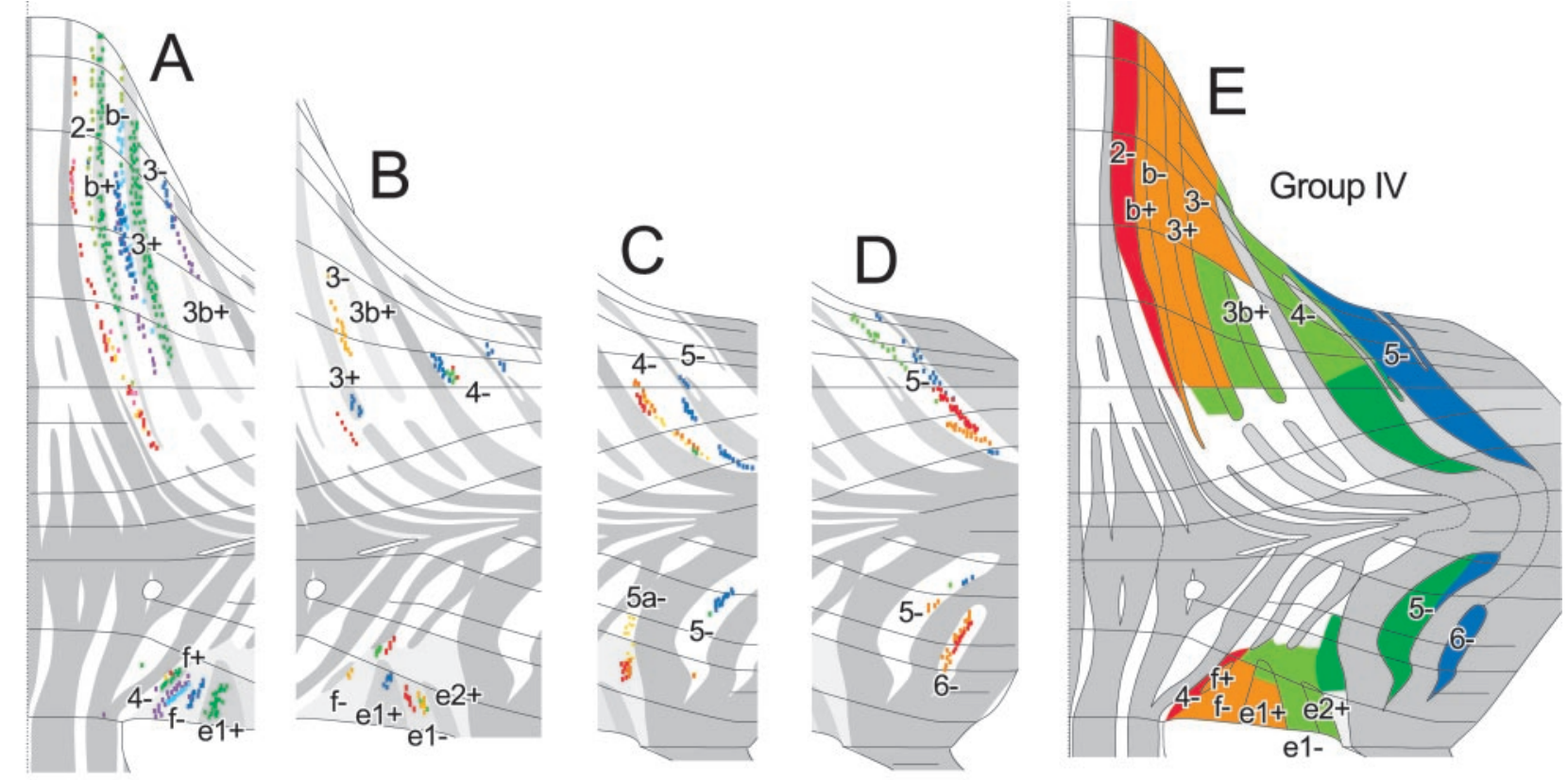

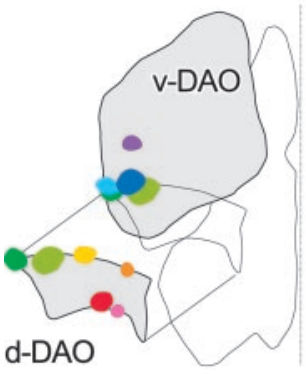

Dorsal view (d-DAO shifted)

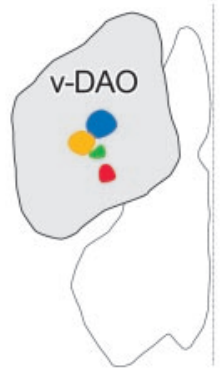

Dorsal view

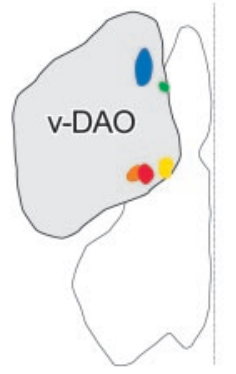

Dorsal view

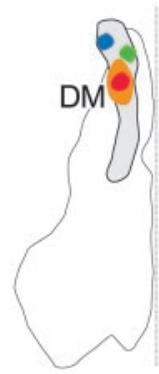

Dorsal view

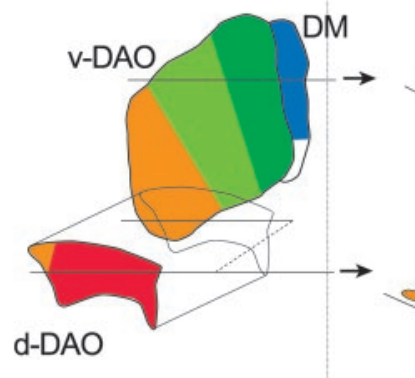

Dorsal view (d-DAO shifted)

Figure 7. Mapping of labeled climbing fibers and summarized topographic scheme of the olivocerebellar projection belonging to group IV. This group consisted of projections from the dorsal fold of the DAO (d-DAO), ventral fold of the DAO (v-DA0), and dorsomedial subnucleus of the P0 (DM) to $2-/ / 4-, b+/ / f+, b-/ / f-, 3+/ / e+, 3-/ / e 1-, 4-/ / 5-$, and $5-/ / 6-$, and possibly to $3 \mathrm{~b}+/ / \mathrm{e} 2+$. A, Plots of labeled climbing fibers in nine injections terminating mainly in compartments $2-/ / 4-, \mathrm{b}+/ / \mathrm{f}+, \mathrm{b}-/ / \mathrm{f}-$, and $3+/ / \mathrm{e}+$. Two injections ( green and light green) were spread to the d-DAO and v-DAO.B, Plots of labeled climbing fibers in four injections in the intermediate v-DA0, terminating in several compartments, including $3-/ / \mathrm{e} 1-$ (yellow). C, Plots of labeled climbing fibers in five injections in the medial v-DA0, terminating mainly in $4-/ / 5-$ and also in caudal $5 a-$. D, Plots of labeled climbing fibers in four injections in the DM, terminating in $5-/ / 6-$ and in caudal 5- Labeled climbing fibers in 9 of 22 experiments were virtually aligned in a single narrow band, if a small number of outside climbing fibers can be ignored. E, Putative olivocerebellar topography within group III determined from the results shown in $A$ and $B$.

tion in the cerebellar cortex within each of the five groups could be mapped to an orientation axis defined in the subnuclei of the inferior olive (Fig. 8). These axes may be related to the conjunctive relationship among different subnuclei and the convoluted organization of the entire inferior olive, although the significance of these axes in the evolution and development has to be clarified.

In the part of the inferior olive that belongs to group I (Fig. 4), the orientation axis ran from subnucleus a of the MAO (caudal to rostral), through the r-MAO (caudal to rostral) and v-PO (medial to lateral), to the d-PO (lateral to medial) (Fig. $8 \mathrm{~A}$, green arrows). As shown in the three-dimensional display of olivary subnuclei, these areas of the inferior olive were located close to each other along the axis.

Group II (Fig. 5) had a slightly more complex topography but could be reasonably explained by assuming two axes. The first orientation axis ran from the caudal subnucleus $\beta$ and caudal subnucleus $\mathrm{c}$, which are cytologically indistinguishable from each other (Ruigrok and Cella, 1995), through the DMCC and the caudal DM, to the caudomedial v-PO (Fig. $8 \mathrm{~A}$, blue arrows). Although these subnuclei are separated from each other by the white matter, they are still located close to each other along the axis. The second axis ran caudorostrally through the medial and lateral subnucleus $\mathrm{c}$ in succession (Fig. $8 \mathrm{~A}$, violet arrows). Within the compartments that belonged to group II in the cerebellar cortex, the first and second axes corresponded to the rostral and caudal parts $(\mathrm{a}+/ / 2+$ in lobules V-VIa and VIII-X and $2 \mathrm{~b}+/ / 4+$ ) (Fig. $8 \mathrm{~A}$, left panel, blue arrow) and the central parts $(\mathrm{a}+/ / 2+$ in lobules VIb-c and VII and $c+/ / 4 b+$ and $d+/ / 5 a+)$ (Fig. 8 A, left panel, violet arrow), respectively.

In group III (Fig. 6), the topography could be explained rather simply because only subnucleus b of the MAO was involved. The orientation axis first ran in the lateral subnucleus $b$ (caudal to rostral), corresponding to the projection to $1-(\mathrm{med}) / / 1-$, $1-($ lat $) / a-/ / 2-$, and $2 a-/ / 3-$. The axis then ran in the medial subnucleus b (again caudal to rostral), corresponding to the projection to $2 \mathrm{~b}-/ / 4 \mathrm{a}-, \mathrm{c}-/ / 4 \mathrm{~b}-$, and $\mathrm{d}-/ / 5 \mathrm{a}-$ (Fig. $8 \mathrm{~A}$, ocher arrows).

In group IV (Fig. 7), a single orientation axis running from the d-DAO (medial to lateral), through the v-DAO (caudolateral to 


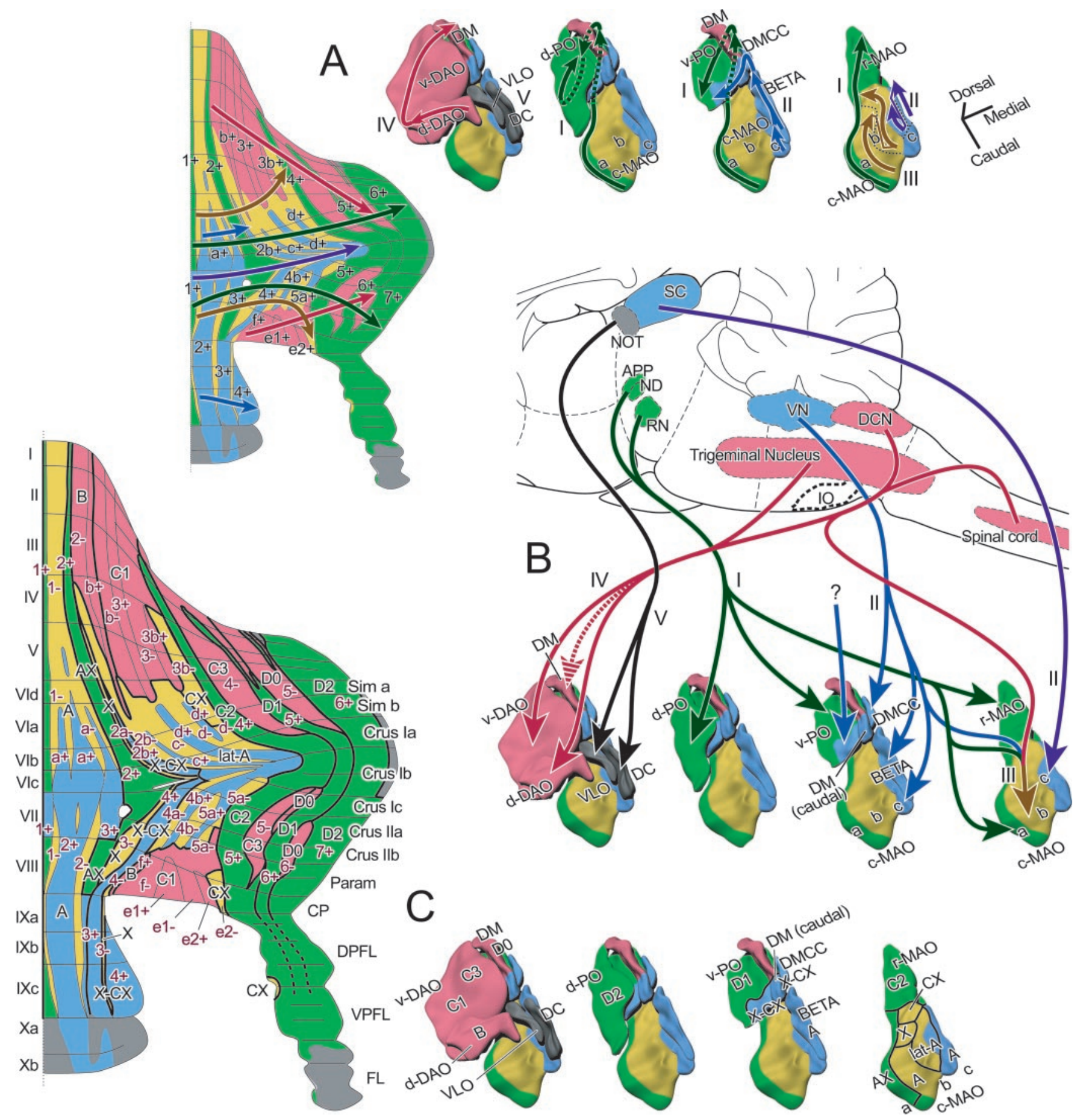

Figure 8. Organization of aldolase C compartments and topographic olivocerebellar projection based on the five groups in the present study. A, Topographic organization explained with orientation axes. Areas belonging to each of the five groups (green, blue, yellow, red, and gray for groups I-V, respectively) were mapped on the aldolase ( compartment pattern of the cerebellar cortex (left) and on the solid schemes of the inferior olive (right). Arrows with dark colors indicate the mediolateral direction in each group in the cerebellar cortex and the orientation axes in the inferior olive. The orientation axes correspond to the medial to lateral direction in the cerebellar cortex in terms of the topography of the projection. Group II has two orientation axes, corresponding to the projection to the central cortical area from the rostral and lateral subnucleus c (violet) and to the projection to the rostral and caudal cortical areas from the caudal subnucleus $C$, subnucleus $\beta$, DMCC, caudal dorsomedial subnucleus of the PO (DM), and caudomedial ventral lamella of the PO (v-P0) (blue). This panel is based on the data shown in Figures $4-7$. Group V (gray) consists of the flocculus and nodulus and a few areas in the hemisphere innervated by the DC and VLO (Sugihara et al., 2004). B, A schematic of major inputs to the inferior olive reported so far sorted according to the groups classified in the present study. Question mark indicates that the specific inputs in those areas have not been clarified. See Discussion for details. Origins of major afferents to the inferior olive are arbitrarily colored. APP, Area parafascicularis prerubralis; DCN, dorsal column nucleus; 10, inferior olive; ND, nucleus of Darkschewitsch; NOT, nucleus of the optic tract; RN, red nucleus; SC, superior colliculus; VN, vestibular nucleus. C, Correspondence between the aldolase C-based five-group olivocerebellar organization in the present study and conventional olivocerebellar zones (zones A-D). Colors indicate the same groups as in panels $A$ and $B . I-X$, Lobules I-X; $a-c$, sublobules a - cor subnuclei a- $c$; BETA, subnucleus $\beta$; $C-M A 0$, caudal part of the MA0; $C P$, copula pyramidis; $\mathrm{Cr}$ I, crus I of ansiform lobule; $C \mathrm{Cll}$, crus II of ansiform lobule; $d-D A 0$, dorsal fold of the DAO; $d-P 0$, dorsal lamella of the PO; DC, DC of Kooy; DM, dorsomedial subnucleus of the PO; DMCC, DMCC subnucleus; DPFL, dorsal paraflocculus; FL, flocculus; Param, paramedian lobule; r-MAO, rostral part of the MAO; Sim, simple lobule; v-DA0, ventral fold of the DAO; v-PO, ventral lamella of the PO; VPFL, ventral paraflocculus. 
Table 2. Major olivocerebellar topographic projection pattern in terms of aldolase C compartments and their putative correspondence to classic olivocerebellar zones

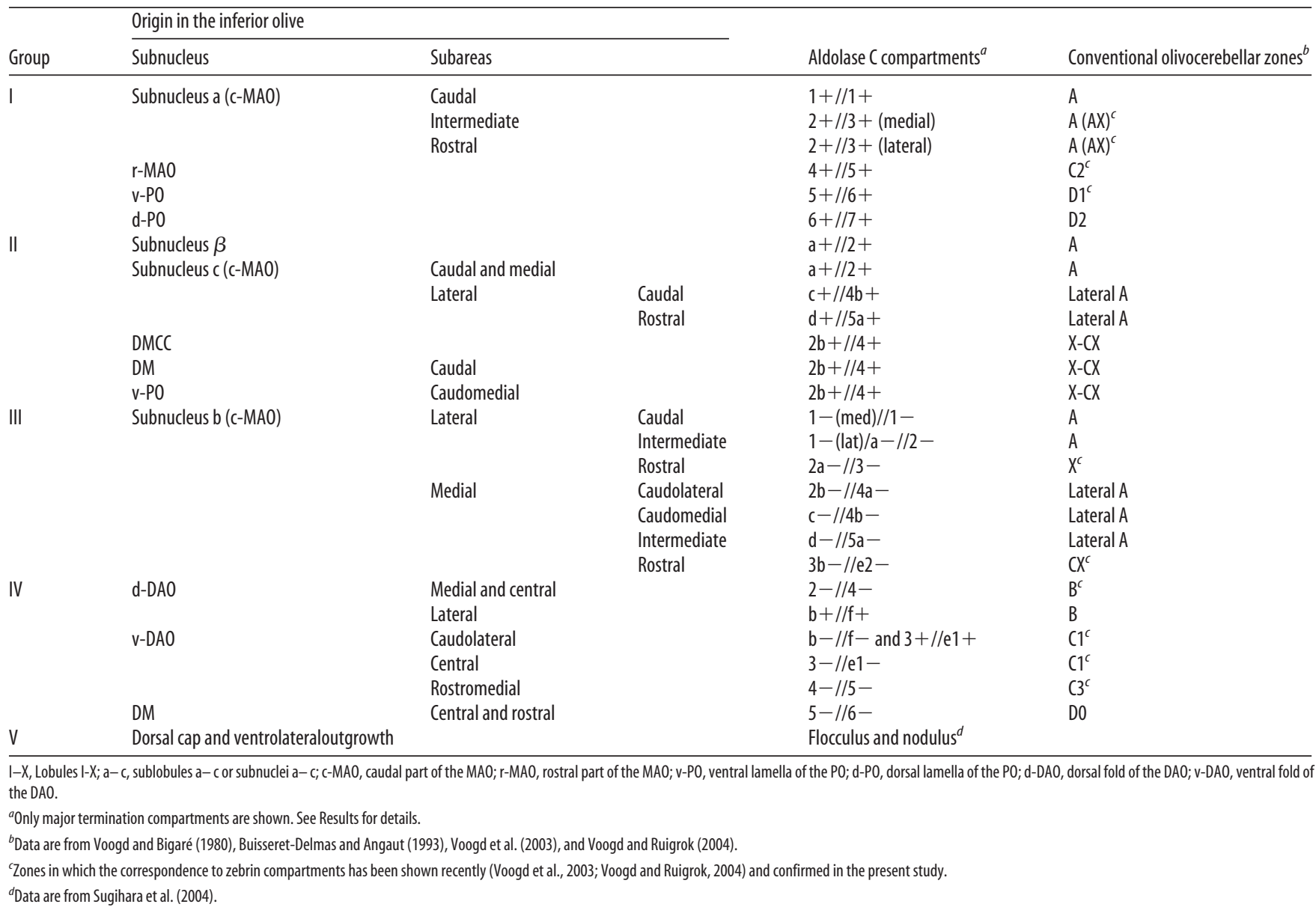

rostromedial), to the DM could mostly account for the topographical projection to all compartments (medial to lateral) in group IV (Fig. $8 \mathrm{~A}$, red arrows). These subnuclei of the inferior olive are located close to each other along the axis.

Concerning group V, we examined the detailed projection pattern from the DC and VLO to the flocculus (Sugihara et al., 2004). However, it was not easy to determine an orientation axis in this group.

\section{Discussion}

This study showed that the organization of the olivocerebellar topography and aldolase $\mathrm{C}$ compartment pattern is rather simply explained in the five-group scheme, although the significance of aldolase C expression itself is not clear (cf. Welsh et al., 2002). Whether the corticonuclear and olivonuclear topographies are explained according to this five-group organization will be the next question.

\section{Organization of the inferior olive: five groups related to distinct functions}

The divisions of the inferior olive in the present five-group scheme do not fully correspond to the conventional olivary subnuclei. Histochemical and immunohistological observations have recognized molecular patterns in the olive that are not necessarily correlated with these subdivisions [acetylcholinesterase (Marani et al., 1977); calbindin calcitonin gene-related peptide and parvalbumin (Wassef et al., 1992)]. The present five groups and the olivary molecular patterns may agree in the c-MAO (Wassef et al., 1992) but not in other areas of the olive. In con- trast, the present grouping in the olive seems to correlate well with the difference in input to the olive.

To consider the functional aspects of the groups defined here, major inputs to the parts of the inferior olive were compared using previous reports (Fig. $8 \mathrm{~B}$ ). In group I, the v-PO, d-PO, $\mathrm{r}-\mathrm{MAO}$, and subnucleus a receive input from the cerebral cortex relayed by nuclei in the mesodiencephalic junction (Swenson and Castro, 1983; Onodera, 1984; Holstege and Tan, 1988; de Zeeuw, 1989) (Fig. 8 B, green arrows), although the subnucleus a also receive input from the spinal cord (Matsushita et al., 1991).

Concerning group II, the superior colliculus projects to what seems to be the medial and lateral subnucleus $\mathrm{c}$ of the MAO, and the vestibular nuclei project to the DMCC, subnucleus $\beta$, and subnucleus c (Brown et al., 1977; Swenson and Castro, 1983; Gerrits et al., 1985; Akaike, 1992) (Fig. 8 B, violet and blue arrows). The projections from the spinal cord and dorsal column nucleus, which are predominant in the subnucleus b, lack in the subnucleus $c$ and $\beta$ (Boesten and Voogd, 1975). The major inputs to the caudal DM and caudomedial v-PO are unknown.

Concerning group III, the subnucleus b of the MAO receives heterogeneous input from the spinal cord and dorsal column nuclei with crude somatotopy (Boesten and Voogd, 1975; Berkley and Hand, 1978; Matsushita et al., 1992; Molinari et al., 1996) and from the vestibular nuclei and the mesodiencephalic junction (Swenson and Castro, 1983; Onodera, 1984; Gerrits et al., 1985) (Fig. $8 B$, ocher arrow).

The d-DAO and v-DAO in group IV receive inputs from the spinal cord and the dorsal column nuclei with refined cutaneous 
somatotopy (v-DAO) and with preponderance of deep modality (d-DAO) (Berkley and Hand, 1978; Gellman et al., 1983; Swenson and Castro, 1983; Matsushita et al., 1992; Molinari et al., 1996) (Fig. 8B, red arrows). Input to the DM has not been reported clearly. However, some figures in the reports by Swenson and Castro (1983, their Fig. 5-3), Huerta et al. (1985, their Fig. 2), and Van Ham and Yeo (1992, their Figs. 4, 5) suggest the trigeminal projection to the DM (Fig. $8 \mathrm{~B}$, hashed red arrow).

The VLO and DC subnuclei, which belong to group V, receive input from nuclei of the accessory optic system (Leonard et al., 1988) (Fig. 8 B, black arrows).

Thus, groups I-V primarily receive mesodiencephalic, vestibular and collicular, somatosensory plus other (mesodiencephalic and vestibular), somatosensory, and retinal inputs, respectively. This finding indicates that the aldolase C labeling pattern is closely related to the functional compartmentalization of the cerebellar cortex. However, it does not mean each aldolase $\mathrm{C}$ compartment simply circumscribes functionally homogeneous climbing fibers, because climbing fibers originating from a small group of inferior olive neurons are distributed in a longitudinal band-shaped area that is much narrower than a single aldolase $\mathrm{C}$ compartment (Fig. 3A). Therefore, the relationship between the aldolase $\mathrm{C}$ compartments and the electrophysiologically identified longitudinal structure in the cerebellar cortex regarding climbing fiber activity (Ekerot et al., 1991; Lang et al., 1999; Hanson et al., 2000) is to be examined (cf. Voogd et al., 2003).

\section{Interpretation of the conventional cerebellar zones with the present results}

Previous retrograde and anterograde mass labeling studies and electrophysiological studies have identified so-called conventional olivocerebellar zones (A, B, X, C1, CX, X-CX, C2, C3, D0, $\mathrm{D} 1$, and D2) that are innervated topographically from different subnuclei of the inferior olive (Groenewegen and Voogd, 1977; Brodal, 1981; Azizi and Woodward, 1987; Buisseret-Delmas and Angaut, 1993). A relationship between conventional olivocerebellar zones and zebrin (aldolase C) compartments has been shown recently in several areas that mainly belong to groups I and IV in our designation (Voogd et al., 2003, 2004), which mostly agrees with the present results (see the last note of Table 2). However, concerning compartments belonging to groups II and III, which are rather complicated than other compartments, their rostrocaudal continuity or the olivocerebellar projections to them could not be properly analyzed in previous studies. The relationship between conventional olivocerebellar zones and aldolase $\mathrm{C}$ compartments can be determined throughout the cerebellar cortex in this study by comparing the present results with a scheme of the conventional olivocerebellar topography in terms of the positions of zones and olivary origins, as listed in Table 2 and depicted in Figure $8 C$.

Conventional zone A occupies nearly the entire vermis, and lateral zone A extends to the central pars intermedia. Although these zones presumably include multiple aldolase C compartments, its subdivisions have not been much clarified, except for the most lateral part of zone A (recently designated as AX) that corresponds to $2+/ / 3+$ (Voogd and Ruigrok, 2004). The present results show clear subdivisions corresponding to all aldolase $\mathrm{C}$ compartments within conventional zone A and lateral zone A. These subdivisions belong to groups I, II, and III. The separate entity of lateral zone A proposed on the basis of the corticonuclear projection pattern (Goodman et al., 1963) was supported, because the lateral subnucleus $\mathrm{c}$ and the neighboring medial subnucleus b project to it (Fig. 8C).
Conventional zones $\mathrm{B}, \mathrm{C} 1, \mathrm{C} 3$, and D0 belong to group IV according to the present results. Zones $\mathrm{B}$ and $\mathrm{C} 1$ appear to correspond to multiple negative and lightly positive aldolase $\mathrm{C}$ compartments in the rostral and caudal cerebellar cortex (Table 2, Fig. $8 C)$. Zone C3 mostly corresponds to $4-/ / 5-$. Zone C2 mostly corresponds to $4+/ / 5+$ and extends into the paraflocculus and the most caudal portion of the flocculus (Ruigrok et al., 1992; Sugihara et al., 2004). These results concerning zones B, C1, C2, and C3 were similar to the recent observations of Voogd et al. (2003) and Voogd and Ruigrok (2004). Zone C2 in lobule IX (Sato and Barmack, 1985; Voogd and Ruigrok, 1997) was not confirmed in the present study.

Based on the present results, zone D1 corresponds to $5+/ / 6+$ innervated by the $\mathrm{v}-\mathrm{PO}$, and lateral zone D2 corresponds to $6+/ / 7+$ innervated by the $\mathrm{d}-\mathrm{PO}$. This projection is the same as that postulated in the cat (Voogd and Bigaré, 1980; Rosina and Provini, 1982) and inferred in the rat (Voogd et al., 2004) but is not consistent with previous reports in the rat (Azizi and Woodward, 1987; Buisseret-Delmas and Angaut, 1993), in which the $\mathrm{v}-\mathrm{PO}$ has been thought to innervate a more lateral area than the d-PO. Zone D0 has been reported to be located medial to D1 (Buisseret-Delmas and Angaut, 1993) or between D1 and D2 (Voogd et al., 2003). The present results suggest that D0 is located not only in the entire 5-//6- compartment but also in a small lateral part of the $4-/ / 5-$ compartment in crus II, indicating that both reports are correct, although the nuclear projections of these areas have to be determined to confirm it.

Among zones added later (X, CX, X-CX) (Buisseret-Delmas et al., 1993; Yatim et al., 1995), zone X likely corresponds to 2a-// $3-$, and zone CX corresponds to $3 \mathrm{~b}-/ / \mathrm{e} 2-$ (parts of group III). Zone X-CX remains enigmatic but appears to correspond to $2 \mathrm{~b}+/ / 4+$ innervated by the DMCC. No zones previously reported appear to correspond to the projection of the caudal DM and caudomedial v-PO to the rest of $2+/ / 4+$. However, this projection may also be regarded as part of zone $\mathrm{X}-\mathrm{CX}$ because it resembles the projection of the DMCC.

Besides zones A-D, more classical aspects of the cerebellar organization can be related to the present results. Group II is located only caudal to the primary fissure, and group IV occupies a substantial portion of the area rostral to the primary fissure. This may help explain the apparent difference in the gross function of the cerebellum rostral and caudal to the primary fissure (Brodal, 1981), although mossy fiber projection must also be considered.

Functional implications of the link between the specific rostral and caudal aldolase $\mathrm{C}$ compartments and the translobular branching of single olivocerebellar axons (Sugihara et al., 2001) as the basis for this link are not known. However, the rostrocaudal boundary defined here seems a fundamental landmark at least for the morphological organization of the cerebellar cortex.

\section{References}

Ahn AH, Dziennis S, Hawkes R, Herrup K (1994) The cloning of zebrin II reveals its identity with aldolase C. Development 120:2081-2090.

Akaike T (1992) The tectorecipient zone in the inferior olivary nucleus in the rat. J Comp Neurol 320:398-414.

Andersson G, Oscarsson O (1978) Climbing fiber microzones in cerebellar vermis and their projection to different groups of cells in the lateral vestibular nucleus. Exp Brain Res 32:565-579.

Azizi SA, Woodward DJ (1987) Inferior olivary nuclear complex of the rat: morphology and comments on the principles of organization within the olivocerebellar system. J Comp Neurol 263:467-484.

Bailly Y, Schoen SW, Delhaye-Bouchaud N, Kreutzberg GW, Mariani J (1995) 5'-Nucleotidase activity as a synaptic marker of parasagittal compartmentation in the mouse cerebellum. J Neurocytol 24:879-890. 
Berkley KJ, Hand PJ (1978) Projections to the inferior olive of the cat. II. Comparisons of input from the gracile, cuneate and the spinal trigeminal nuclei. J Comp Neurol 180:253-264.

Boesten AJ, Voogd J (1975) Projections of the dorsal column nuclei and the spinal cord on the inferior olive in the cat. J Comp Neurol 161:215-237.

Brochu G, Maler L, Hawkes R (1990) Zebrin II: a polypeptide antigen expressed selectively by Purkinje cells reveals compartments in rat and fish cerebellum. J Comp Neurol 291:538-552.

Brodal A (1981) Neurological anatomy in relation to clinical medicine, Ed 3. New York: Oxford UP.

Brown JT, Chan-Palay V, Palay SL (1977) A study of afferent input to the inferior olivary complex in the rat by retrograde axonal transport of horseradish peroxidase. J Comp Neurol 176:1-22.

Buisseret-Delmas C, Angaut P (1993) The cerebellar olivocorticonuclear connections in the rat. Prog Neurobiol 40:63-87.

Buisseret-Delmas C, Yatim N, Buisseret P, Angaut P (1993) The X zone and CX subzone of the cerebellum in the rat. Neurosci Res 16:195-207.

de Zeeuw CI, Holstege JC, Ruigrok TJ, Voogd J (1989) Ultrastructural study of the GABAergic, cerebellar, and mesodiencephalic innervation of the cat medial accessory olive: anterograde tracing combined with immunocytochemistry. J Comp Neurol 284:12-35.

Doré L, Jacobson CD, Hawkes R (1990) Organization and postnatal development of zebrin II antigenic compartmentation in the cerebellar vermis of the grey opossum, Monodelphis domestica. J Comp Neurol 291:431-449.

Ekerot C-F, Larson B (1982) Branching of olivary axons to innervate pairs of sagittal zones in the cerebellar anterior lobe in the cat. Exp Brain Res 48:185-198.

Ekerot C-F, Garwicz M, Schouenborg J (1991) Topography and nociceptive receptive fields of climbing fibres projecting to the cerebellar anterior lobe in the cat. J Physiol (Lond) 441:257-274.

Gellman R, Houk JC, Gibson AR (1983) Somatosensory properties of the inferior olive of the cat. J Comp Neurol 215:228-243.

Gerrits NM, Voogd J, Magras IN (1985) Vestibular afferents of the inferior olive and the vestibulo-olivo-cerebellar climbing fiber pathway to the flocculus in the cat. Brain Res 332:325-336.

Goodman DC, Hallett RE, Welch RB (1963) Patterns of localization in the cerebellar corticonuclear projections of albino rat. J Comp Neurol 121:51-67.

Gravel C, Eisenman LM, Sasseville R, Hawkes R (1987) Parasagittal organization of the rat cerebellar cortex: direct correlation between antigenic Purkinje cell bands revealed by mabQ113 and the organization of the olivocerebellar projection. J Comp Neurol 265:294-310.

Groenewegen HJ, Voogd J (1977) The parasagittal zonation within the olivocerebellar projection. I. Climbing fiber distribution in the vermis of the cat cerebellum. J Comp Neurol 174:417-488.

Gwyn DG, Nicholson GP, Flumerfelt BA (1977) The inferior olivary nucleus of the rat: a light and electron microscopic study. J Comp Neurol 174:489-520.

Hanson CL, Chen G, Ebner TJ (2000) Role of climbing fibers in determining the spatial patterns of activation in the cerebellar cortex to peripheral stimulation: an optical imaging study. Neuroscience 96:317-331.

Hawkes R (1997) An anatomical model of cerebellar modules. Prog Brain Res 114:39-52.

Hawkes R, Leclerc N (1987) Antigenic map of the rat cerebellar cortex: the distribution of parasagittal bands as revealed by monoclonal antiPurkinje cell antibody mobA113. J Comp Neurol 256:29-41.

Herrup K, Kuemerle B (1997) The compartmentalization of the cerebellum. Annu Rev Neurosci 20:61-90.

Holstege G, Tan J (1988) Projections from the red nucleus and surrounding areas to the brainstem and spinal cord in the cat. An HRP and autoradiographical tracing study. Behav Brain Res 28:33-57.

Huerta MF, Hashikawa T, Gayoso MJ, Harting JK (1985) The trigemino-olivary projection in the cat: contributions of individual subnuclei. 241:180-190.

Lang EJ, Sugihara I, Welsh JP, Llinas R (1999) Patterns of spontaneous purkinje cell complex spike activity in the awake rat. J Neurosci 19:2728-2739.

Leonard CS, Simpson JI, Graf W (1988) Spatial organization of visual messages of the rabbit's cerebellar flocculus. I. Topology of inferior olive neurons of the dorsal cap of Kooy. J Neurophysiol 60:2073-2090.

Llinás R, Baker R, Sotelo C (1974) Electrotonic coupling between neurons in cat inferior olive. J Neurophysiol 37:560-571.

Marani E, Voogd J, Boekee A (1977) Acetylcholinesterase staining in subdivisions of the cat's inferior olive. J Comp Neurol 174:209-226.

Matsushita M, Ragnarson B, Grant G (1991) Topographic relationship be- tween sagittal Purkinje cell bands revealed by monoclonal antibody to zebrin I and spinocerebellar projections arising from the central cervical nucleus in the rat. Exp Brain Res 84:133-141.

Matsushita M, Yaginuma H, Tanami T (1992) Somatotopic termination of the spino-olivary fibers in the cat, studied with the wheat germ agglutininhorseradish peroxidase technique. Exp Brain Res 89:397-407.

Molinari HH, Schultze KE, Strominger NL (1996) Gracile, cuneate, and spinal trigeminal projections to inferior olive in rat and monkey. J Comp Neurol 375:467-480.

Mukai T, Yatsuki H, Masuko S, Arai Y, Joh K, Hori K (1991) The structure of the brain-specific rat aldolase $\mathrm{C}$ gene and its regional expression. Biochem Biophys Res Commun 174:1035-1042.

Onodera S (1984) Olivary projections from the mesodiencephalic structures in the cat studied by means of axonal transport of horseradish peroxidase and tritiated amino acids. J Comp Neurol 227:37-49.

Ozol K, Hayden JM, Oberdick J, Hawkes R (1999) Transverse zones in the vermis of the mouse cerebellum. J Comp Neurol 412:95-111.

Rosina A, Provini L (1982) Longitudinal and topographical organization of the olivary projection to the cat ansiform lobule. Neuroscience 7:2657-2676.

Ruigrok TJH, Cella F (1995) Precerebellar nuclei and red nucleus. In: The rat nervous system, Ed 2 (Paxinos G, ed), pp 277-308. San Diego: Academic.

Ruigrok TJH, Osse RJ, Voogd J (1992) Organization of inferior olivary projections to the flocculus and ventral paraflocculus of the rat cerebellum. J Comp Neurol 316:129-150.

Sato Y, Barmack NH (1985) Zonal organization of olivocerebellar projections to the uvula in rabbits. Brain Res 359:281-292.

Sugihara I, Wu H-S, Shinoda Y (1999) Morphology of single olivocerebellar axons labeled with biotinylated dextran amine in the rat. J Comp Neurol 414:131-148.

Sugihara I, Wu H-S, Shinoda Y (2001) The entire trajectories of single olivocerebellar axons in the cerebellar cortex and their contribution to cerebellar compartmentalization. J Neurosci 21:7715-7723.

Sugihara I, Ebata S, Shinoda Y (2004) Functional compartmentalization in the flocculus and the ventral dentate and dorsal group y nuclei: An analysis of single olivocerebellar axonal morphology. J Comp Neurol 470:113-133.

Swenson RS, Castro AJ (1983) The afferent connections of the inferior olivary complex in rats. An anterograde study using autoradiographic and axonal degeneration techniques. Neuroscience 8:259-275.

Van Ham JJ, Yeo CH (1992) Somatosensory trigeminal projections to the inferior olive, cerebellum and other precerebellar nuclei in rabbits. Eur J Neurosci 4:302-317.

Voogd J (1967) Comparative aspects of the structure and fibre connexions of the mammalian cerebellum. In: Progress in brain research, Vol. 25: The cerebellum (Fox CA, Snider RS, eds), pp 94-135. Amsterdam: Elsevier.

Voogd J, Bigaré F (1980) Topographical distribution of olivary and cortico nuclear fibers in the cerebellum: a review. In: The inferior olivary nucleus: anatomy and physiology (Courville J, de Montigny C, Lamarre Y, eds), pp 207-234. New York: Raven.

Voogd J, Ruigrok TJH (1997) Transverse and longitudinal patterns in the mammalian cerebellum. Prog Brain Res 114:21-37.

Voogd J, Ruigrok TJH (2004) The organization of the corticonuclear and olivocerebellar climbing fiber projections to the rat cerebellar vermis: the congruence of projection zones and the zebrin pattern. J Neurocytol 33:5-21.

Voogd J, Pardoe J, Ruigrok TJH, Apps R (2003) The distribution of climbing and mossy fiber collateral branches from the copula pyramidis and the paramedian lobule: congruence of climbing fiber cortical zones and the pattern of zebrin banding within the rat cerebellum. J Neurosci 23:4645-4656.

Wassef M, Cholley B, Heizmann CW, Sotelo C (1992) Development of the olivocerebellar projection in the rat: II. Matching of the developmental compartmentations of the cerebellum and inferior olive through the projection map. J Comp Neurol 323:537-550.

Welsh JP, Yuen G, Placantonakis DG, Vu TQ, Haiss F, O’Hearn E, Molliver ME, Aicher SA (2002) Why do Purkinje cells die so easily after global brain ischemia? Aldolase C, EAAT4, and the cerebellar contribution to posthypoxic myoclonus. Adv Neurol 89:331-359.

Wu H-S, Sugihara I, Shinoda Y (1999) Projection patterns of single mossy fibers originating from the lateral reticular nucleus in the rat cerebellar cortex and nuclei. J Comp Neurol 411:97-118.

Yatim N, Compoint C, Buisseret P, Angaut P, Buisseret-Delmas C (1995) On the caudal extension of the $\mathrm{X}$ zone in the cerebellar cortex of the rat. Neurosci Res 23:223-227. 